\title{
A light-fronts approach to electron-positron pair production in ultra-relativistic heavy-ion collisions
}

\author{
B. Segev and J. C. Wells* \\ Institute for Theoretical Atomic and Molecular Physics, \\ Harvard-Smithsonian Center for Astrophysics, \\ 60 Garden Street, Cambridge, MA 02138
}

(February 11, 2018)

\begin{abstract}
We perform a gauge-transformation on the time-dependent Dirac equation describing the evolution of an electron in a heavy-ion collision to remove the explicit dependence on the long-range part of the interaction. We solve, in an ultra-relativistic limit, the gauged-transformed Dirac equation using lightfront variables and a light-fronts representation, obtaining non-perturbative results for the free pair-creation amplitudes in the collider frame. Our result reproduces the result of second-order perturbation theory in the small charge limit while non-perturbative effects arise for realistic charges of the ions.

PACS number: 34.50.-s, 25.75.-q, 11.80.-m, 12.20.-m
\end{abstract}

*New address: Oak Ridge National Laboratory, P.O. Box 2008, Oak Ridge, TN 37831-6203 


\section{INTRODUCTION}

Electron-positron pair production from the vacuum in strong electromagnetic fields is a fundamental prediction of QED [1] 3]. In heavy-ion collisions at energies near the Coulomb barrier, quasi-bound molecular states are formed with binding energies which dive into the negative-energy continuum, resulting in a resonance which decays into an electronpositron pair [2, 1 , 8]. In contrast, for ultra-relativistic heavy-ion collisions at peripheral impact parameters, the ions execute straight-line trajectories and reside near each other for only a very short time. The high charge of the individual ions and the strong Lorentz contraction combine to produce fields sufficient for electromagnetic pair production through a qualitatively different process. Large cross sections for electro-magnetic pair production in these collisions were theoretically predicted [9] and experimentally observed [10 17].

In lowest-order perturbation theory, the amplitudes for pair production in heavy-ion collisions are calculated from two-photon exchange diagrams [18. The quantum field theoretical treatment of this process was reduced to a classical-field approach [19]. Experimental observations of free pair production in the energy range around ten $\mathrm{GeV}$ per nucleon (in the collider reference frame) are in agreement with second-order perturbation theory [10, 12, 14, 16]. For lower energies of a few $\mathrm{GeV}$ per nucleon (collider frame), experimental results for free and bound-free pair production show deviations from the predictions obtained from two-photon exchange diagrams [11,13. This is likely due to two-center Coulomb effects [20,21]. In the near future, larger ultra-relativistic energies above one hundred $\mathrm{GeV}$ per nucleon (collider frame) will be available. New non-perturbative effects may become important at collidingbeam accelerators such as the Relativistic Heavy-ion Collider (RHIC) at Brookhaven and

possibly the Large Hadron Collider (LHC) at CERN. These non-perturbative effects are the subject of our present work.

Previous theoretical works on high-energy non-perturbative effects considered unitarity violation in lowest-order perturbation theory, multiple-pair production, and corrections to production cross sections [22 32]. An open question, crucial for the beam stability, is 
whether the non-perturbative effects will enhance or reduce the cross section for bound-free production, i.e. production with capture [30,32].

In a non-perturbative treatment, starting from the QED Lagrange density operator, the Euler-Lagrange equations of motion for the quantum fields are equivalent, under physical assumptions, to the one-particle Dirac equation interacting with classical, electromagnetic fields [33]. Calculations of probabilities and correlations can then be reduced to solving the two-center time-dependent Dirac equation, which describes the dynamics of an electron in the classical field of two relativistically moving charges.

In the ultra-relativistic limit, the ions are practically moving at the speed of light. The classical electromagnetic field of a massless charged particle was studied in Ref. [34]. It can be described by pure gauge potentials, with different gauges in different regions of space-time. The eikonal approximation was then reproduced from an exact solution of a quantum-mechanical equation in this field. A similar approach was recently used in targetframe calculations of bound-free pair production, where a gauge transformation was used to remove the long-range Coulomb effects [29.32].

The use of gauge transformations is fundemental to these calculations. The term gauge transformation, as used here, is not to be confused with a gauge-symmetry transformation in which both the wave function and the fields are transformed so as to keep the equation of motion invariant. Here, as well as in Refs. [24,35,36], for example, a space-time dependent phase is used to transform either the wave function or the fields in order to obtain a different equation of motion. The connection between the solution of the original problem and the gauge-transformed problem depends on the asymptotic (infinite time) behavior of the gauge function employed; i.e. on the induced changes to the initial and final states [24, 35, 36]. Gauge transformations should also be applied with care when used in calculations employing truncated basis sets 3537 .

In this work, a gauge transformation is used to solve the two-center Dirac equation describing an electron during a relativistic heavy-ion collision. A closed form expression for the pair-production amplitudes in the ultra-relativistic limit is found. First, in section II, 
the ultra-relativistic limit for the two-center Dirac equation is obtained and discussed. In section III, exact and asymptotic relations between the physical amplitudes and the gauged transformed ones are established. In section IV, the light-fronts representation is introduced and the foundations are laid for the construction of the exact solution in section $\mathrm{V}$, where the perturbative limit is considered as well. The physical contents of our results and an outlook for future applications are finally considered in section VI. Details of some derivations are given in appendices.

\section{AN ULTRA-RELATIVISTIC LIMIT TO THE TWO-CENTER TIME-DEPENDENT DIRAC EQUATION}

Consider a collision between two ions with charges $Z_{A}$ and $Z_{B}$ and velocities $\beta \hat{z}$ and $-\beta \hat{z}$, respectively, moving parallel to each other at an impact parameter of $2 \vec{b}$ (see Fig. 11). An external-field approach to the influence of these ions on the vacuum is appropriate for peripheral impact parameters (i.e. no nuclear interactions), heavy ions, and ultrarelativistic energies, when to a very good approximation, the ions continue intact on their parallel, straight-line trajectories. The two-center Dirac equation for an electron in the time-dependent external field of these ions is given by:

$$
i \frac{\partial}{\partial t}|\Phi(\vec{r}, t)\rangle=\left[\hat{H}_{0}+\hat{H}_{A}(t)+\hat{H}_{B}(t)\right]|\Phi(\vec{r}, t)\rangle
$$

where $|\Phi(\vec{r}, t)\rangle$ is the Dirac spinor wave function of the electron, $\hat{H}_{0}$ is the free Dirac Hamiltonian and $\hat{H}_{A}(t)$ and $\hat{H}_{B}(t)$ are each the interaction with one ion,

$$
\begin{aligned}
& \hat{H}_{0} \equiv-i \check{\alpha} \cdot \vec{\nabla}+\check{\gamma}^{0}, \\
& \hat{H}_{A}(t) \equiv\left(\mathrm{I}_{4}-\beta \check{\alpha}_{z}\right) \frac{-Z_{A} \alpha}{\sqrt{\left(\vec{r}_{\perp}-\vec{b}\right)^{2} / \gamma^{2}+(z-\beta t)^{2}}}, \\
& \hat{H}_{B}(t) \equiv\left(\mathrm{I}_{4}+\beta \check{\alpha}_{z}\right) \frac{-Z_{B} \alpha}{\sqrt{\left(\vec{r}_{\perp}+\vec{b}\right)^{2} / \gamma^{2}+(z+\beta t)^{2}}} .
\end{aligned}
$$

We are working in the collider frame, using natural units $\left(c=1, m_{e}=1\right.$, and $\left.\hbar=1\right)$,

and applying the conventional notation; $\beta \equiv v / c, \gamma \equiv 1 / \sqrt{1-\beta^{2}}$. $\alpha$ is the fine-structure 
constant, $\check{\alpha}$ and $\check{\gamma}^{\mu}$ are Dirac matrices in the Dirac representation, as in Ref. [四]; $\check{\sigma}$ are the Pauli matrices; and $\mathrm{I}_{2}, \mathrm{O}_{2}, \mathrm{I}_{4}$, and $\mathrm{O}_{4}$ are the 2-dimensional and 4-dimensional unit and zero matrices.

We would like to consider the ultra-relativistic limit in which

$$
\beta \rightarrow 1, \quad \gamma \gg b, r_{\perp}
$$

Eq. (1) does not simplify in this limit in a straight-forward way because, for any given time, the long-range behavior of the interaction terms $\hat{H}_{A}$ and $\hat{H}_{B}$ is independent of $\gamma$ as $z \rightarrow \pm \infty$ (see Fig. 2). A simple ultra-relativistic limit can be obtained by first applying a gauge transformation in order to remove this long-range tail of the interaction. The gaugetransformed wave function $|\Psi(\vec{r}, t)\rangle$ is defined by

$$
\begin{gathered}
|\Psi(\vec{r}, t)\rangle \equiv U(z, t)|\Phi(\vec{r}, t)\rangle \\
U(z, t) \equiv \exp \left\{i Z_{A} \alpha \ln \left[-\gamma(t-z)+\sqrt{b^{2}+\gamma^{2}(t-z)^{2}}\right]\right. \\
\left.+i Z_{B} \alpha \ln \left[+\gamma(t+z)+\sqrt{b^{2}+\gamma^{2}(t+z)^{2}}\right]\right\} .
\end{gathered}
$$

The Dirac equation for $|\Psi(\vec{r}, t)\rangle$ is obtained from Eq. (11),

$$
i \frac{\partial}{\partial t}|\Psi(\vec{r}, t)\rangle=\left[\hat{H}_{0}+\hat{W}_{A}(t)+\hat{W}_{B}(t)\right]|\Psi(\vec{r}, t)\rangle
$$

where the new interaction terms are

$$
\begin{aligned}
\hat{W}_{A}(t)= & \left(\mathrm{I}_{4}-\beta \check{\alpha}_{z}\right) \frac{-Z_{A} \alpha}{\sqrt{\left(\vec{r}_{\perp}-\vec{b}\right)^{2} / \gamma^{2}+(z-\beta t)^{2}}} \\
& -\left(\mathrm{I}_{4}-\check{\alpha}_{z}\right) \frac{-Z_{A} \alpha}{\sqrt{\vec{b}^{2} / \gamma^{2}+(z-t)^{2}}}, \\
\hat{W}_{B}(t)= & \left(\mathrm{I}_{4}+\beta \check{\alpha}_{z}\right) \frac{-Z_{B} \alpha}{\sqrt{\left(\vec{r}_{\perp}+\vec{b}\right)^{2} / \gamma^{2}+(z+\beta t)^{2}}} \\
& -\left(\mathrm{I}_{4}+\check{\alpha}_{z}\right) \frac{-Z_{B} \alpha}{\sqrt{\vec{b}^{2} / \gamma^{2}+(z+t)^{2}}} .
\end{aligned}
$$

Figure 3 demonstrates the short-range character of this gauge-transformed interaction. Similar gauges have been used in Refs. 38,24, which reduce in the limit $\beta \rightarrow 1$ to Eq. (7). Unlike 
Eq. (1), the gauge-transformed equation, Eq. (8), has a simple ultra-relativistic limit [32,34]. In the limits of Eq. (5) (large $\gamma$, small $r_{\perp}$, and small impact parameter $\left.b,\right), \hat{W}_{A}\left(\hat{W}_{B}\right)$ has a sharp, delta-function dependence on $t-z(t+z)$ [32, 34, 39, (see appendix A), i.e.

$$
\begin{aligned}
& \hat{W}_{A} \rightarrow\left(\mathrm{I}_{4}-\check{\alpha}_{z}\right) Z_{A} \alpha \delta(t-z) \ln \left[\frac{\left(\vec{r}_{\perp}-\vec{b}\right)^{2}}{b^{2}}\right], \\
& \hat{W}_{B} \rightarrow\left(\mathrm{I}_{4}+\check{\alpha}_{z}\right) Z_{B} \alpha \delta(t+z) \ln \left[\frac{\left(\vec{r}_{\perp}+\vec{b}\right)^{2}}{b^{2}}\right] .
\end{aligned}
$$

Consider the physical nature of this limit. A $\delta$ function over time alone would indicate a sudden interaction of the ions with the vacuum. In the gauge-transformed equation, Eq. (8), with the interactions of Eqs. (11) and (12), as they move, the ions are continuously interacting with the vacuum. Naturally, this interaction is singular on the trajectories of the ions, as it was before the ultra-relativistic limit has been taken; but an additional singularity is induced in the ultra-relativistic limit by the extreme Lorentz contraction of the field. In this limit, the interaction is infinite on the two planes perpendicular to the ions trajectories, and vanishes elsewhere. In the following, we calculate pair production amplitudes using Eq. (8) with the interactions in Eqs. (11) and (12). The region of large $r_{\perp}$ is not properly accounted for in this treatment, but contributions from this region to pair production are assumed to be small.

The interactions in Eqs. (11) and (12) have zero range in the longitudinal direction and a logarithmic behavior in the transverse direction, similar to the potential of a line of charge. In the limit $\beta \rightarrow 1$, the two ions are moving at the speed of light and thus the interaction planes described above coincide with the light fronts, given by $z= \pm t$ (see Fig. (t). Finally, we note that $\left(\mathrm{I}_{4} \pm \check{\alpha}_{z}\right) / 2$ are orthonormal projection operators. The 4-Dirac spinor wave function of the electron can be decomposed into two orthogonal components,

$$
\begin{aligned}
\left|\Psi_{+}(\vec{r}, t)\right\rangle & \equiv \frac{1}{2}\left(\mathrm{I}_{4}+\check{\alpha}_{z}\right)|\Psi(\vec{r}, t)\rangle \\
\left|\Psi_{-}(\vec{r}, t)\right\rangle & \equiv \frac{1}{2}\left(\mathrm{I}_{4}-\check{\alpha}_{z}\right)|\Psi(\vec{r}, t)\rangle .
\end{aligned}
$$

Each ion interacts directly only with one of these components; $Z_{A}$ with $\left|\Psi_{-}(\vec{r}, t)\right\rangle$ and $Z_{B}$ with $\left|\Psi_{+}(\vec{r}, t)\right\rangle$. 


\section{ASYMPTOTIC SOLUTIONS AND TRANSITION AMPLITUDES}

In scattering theory, characterized by free initial and final states, a complete solution is generally given by the set of asymptotic transition amplitudes between plane waves, (the

S-matrix). In this section, we define the transition amplitudes, $S_{k}^{(j)}$, for the electronic spinor wave function $|\Phi\rangle$, i.e. for Eq. (11), and the transition amplitudes, $A_{k}^{(j)}$, for the gauge transformed wave function $|\Psi\rangle$, i.e. for Eq. (8), using, as usual, initial and final plane wave states. Use of an initial condition of a single plane wave for Eq. (1) is somewhat subtle since the theory is never free due to long-range Coulomb effects [3]. This is an important point when integrating the amplitudes to obtain predictions for physical observables. One should properly account for actual initial and final states for a given experiment.

A complete set of solutions of the free Dirac equation is given by the Dirac plane waves; $\left\{\left|\chi_{p}(\vec{r}, t)\right\rangle\right\}$. Each plane wave is characterized by three continuous and two discrete quantum numbers; namely, the three components of the momentum, $\vec{p}$, the sign of the energy, (we use $\lambda_{p}=0$ for positive energy electrons, $\lambda_{p}=1$ for negative energy electrons,) and the spin, $\left(\left|s_{p}\right\rangle=|+\rangle\right.$ for spin up and $\left|s_{p}\right\rangle=|-\rangle$ for spin down, $) p \equiv\left\{\vec{p}, \lambda_{p}, s_{p}\right\}$. The plane waves satisfy,

$$
\begin{aligned}
& \hat{H}_{0}\left|\tilde{\chi}_{p}(\vec{r})\right\rangle=E_{p}\left|\tilde{\chi}_{p}(\vec{r})\right\rangle, \\
& \left|\chi_{p}(\vec{r}, t)\right\rangle \equiv \exp \left(-i E_{p} t\right)\left|\tilde{\chi}_{p}(\vec{r})\right\rangle \\
& E_{p}=(-1)^{\lambda_{p}} \varepsilon_{p}, \quad \varepsilon_{p} \equiv \sqrt{p^{2}+1},
\end{aligned}
$$

where Eq. (17) is the condition for being on the energy shell. An explicit form is given, for example, by [3], and in our notation by

$$
\begin{aligned}
\left|\tilde{\chi}_{p}(\vec{r})\right\rangle= & \frac{(2 \pi)^{-3 / 2}}{\sqrt{2 \varepsilon_{p}\left(1+\varepsilon_{p}\right)}} \exp (i \vec{r} \cdot \vec{p}) \\
& \left.\left.\times\left(\begin{array}{cc}
0_{2} & -\mathrm{I}_{2} \\
\mathrm{I}_{2} & 0_{2}
\end{array}\right)\left(\begin{array}{c}
\left(1+\varepsilon_{p}\right)\left|s_{p}\right\rangle \\
\vec{\sigma} \cdot \vec{p}
\end{array}\right) s_{p}\right\rangle\right) \\
\equiv & \exp (i \vec{r} \cdot \vec{p})\left|u_{p}\right\rangle .
\end{aligned}
$$


We define the solution $\left|\phi^{(j)}(\vec{r}, t)\right\rangle$ of Eq. (1) by the initial condition,

$$
\lim _{t_{i} \rightarrow-\infty}\left|\phi^{(j)}\left(\vec{r}, t_{i}\right)\right\rangle=\left|\chi_{j}\left(\vec{r}, t_{i}\right)\right\rangle
$$

The asymptotic transition amplitude $S_{k}^{(j)}$ is then given by

$$
S_{k}^{(j)} \equiv \lim _{t_{f} \rightarrow \infty}\left\langle\chi_{k}\left(\vec{r}, t_{f}\right) \mid \phi^{(j)}\left(\vec{r}, t_{f}\right)\right\rangle
$$

where the bra-ket stands, as usual in non-relativistic and non-covariant notation, for integration over all space $\vec{r}$ at a given time. Likewise, $\left|\psi^{(j)}(\vec{r}, t)\right\rangle$ is defined as the solution of Eq. (8) with the initial condition,

$$
\lim _{t_{i} \rightarrow-\infty}\left|\psi^{(j)}\left(\vec{r}, t_{i}\right)\right\rangle=\left|\chi_{j}\left(\vec{r}, t_{i}\right)\right\rangle
$$

and the asymptotic transition amplitude $A_{k}^{(j)}$ is given by

$$
A_{k}^{(j)} \equiv \lim _{t_{f} \rightarrow \infty}\left\langle\chi_{k}\left(\vec{r}, t_{f}\right) \mid \psi^{(j)}\left(\vec{r}, t_{f}\right)\right\rangle
$$

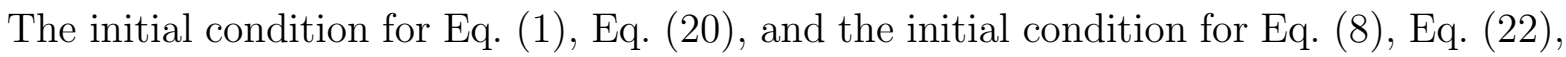
correspond to different initial physical states, as they are not related by the gauge transformation in Eq. (7). A similiar comment is true for the final states used in defining the amplitudes in Eqs. (21) and (23). In general, $S_{k}^{(j)}$ and $A_{k}^{(j)}$ are completely different amplitudes. They are related to each other by the gauge transformation of Eq. (7) in the following way,

$$
\begin{aligned}
S_{k}^{(j)} \equiv & \sum_{p} \sum_{q}\left\langle\chi_{k}\left(\vec{r}, t_{f}\right)\left|U^{\dagger}\left(z, t_{f}\right)\right| \chi_{p}\left(\vec{r}, t_{f}\right)\right\rangle \\
& \times A_{p}^{(q)}\left\langle\chi_{q}\left(\vec{r}, t_{i}\right)\left|U\left(z, t_{i}\right)\right| \chi_{j}\left(\vec{r}, t_{i}\right)\right\rangle,
\end{aligned}
$$

where $\sum_{p}$ stands for integration and summation over all the quantum numbers, $p=$ $\left\{\vec{p}, \lambda_{p}, s_{p}\right\}$. This relation is based on the completeness of the plane-waves basis set and should be questioned if applied with a truncated basis calculation 35 37.

A relation like Eq. (24) holds between any two amplitudes which are related by a gauge transformation. If the gauge transformation $U$ had been otherwise defined so that it would 
become unity for asymptotic times, (i.e. $U \rightarrow 1$ for $t_{i} \rightarrow-\infty$ and $t_{f} \rightarrow \infty$ ), the orthonormality of the plane-waves would eliminate the double sum in Eq. (24), and the asymptotic transition amplitudes $S_{k}^{(j)}$ and $A_{k}^{(j)}$ would be identical. Gauge transformations which share this property have been described as exhibiting asymptotic gauge invariance [36]. Likewise, if the gauge transformation $U$ would be independent of $\vec{r}$ for asymptotic times, (i.e. $U \rightarrow \exp \left(i C_{i}\right)$ for $t_{i} \rightarrow-\infty$, and $U \rightarrow \exp \left(i C_{f}\right)$ for $t_{f} \rightarrow+\infty$, where $C_{i}$ and $C_{f}$ are real numbers), then the double sum in Eq. (24) would again be eliminated and the matrix elements of $U$ in Eq. (24) would contribute only a single constant phase at asymptotic times. In this case, the asymptotic transition probabilities derived from $S_{k}^{(j)}$ and $A_{k}^{(j)}$ would be identical [24].

The specific gauge transformation used here, Eq. (7), is not independent of space at asymptotic times, as is shown in appendix B, and, as a result, does not exhibit asymptotic gauge invariance for the amplitudes or the probabilities derived from them. However, this gauge does have an additional property which relates $S_{k}^{(j)}$ and $A_{k}^{(j)}$ in a way more useful than Eq. (24). It is shown in appendix B that in the special case of symmetric collisions, with $Z_{A}=Z_{B}, S_{k}^{(j)}$ can be expressed as a series expansion in powers of $1 / t_{f}$ and $1 /\left|t_{i}\right|$ whose zero-order term is $A_{k}^{(j)}$.

\section{THE SHARP DIRAC EQUATION IN THE LIGHT-FRONTS REPRESENTATION}

In this section, the sharp Dirac equation, Eq. (8), with the limiting form of the interaction in Eqs. (11), and (12), will be further simplified by changing into light-front variables and by introducing a new representation for the Dirac spinors, the light-fronts representation. This is an appropriate choice of variables and representation, since, in the ultra-relativistic limit of Eq. (5), the interactions are confined to the light fronts. 


\section{A. Definitions and notations}

In terms of light-front variables, space-time and energy-momentum are described by the 4-vectors $\left(\vec{r}_{\perp}, \tau_{+}, \tau_{-}\right)$and $\left(\vec{p}_{\perp}, p_{+}, p_{-}\right)$, where

$$
\begin{aligned}
& \tau_{ \pm} \equiv(t \pm z) / 2 \\
& p_{ \pm} \equiv E_{p} \pm p_{z} \\
& p_{+} p_{-}=1+p_{\perp}^{2}
\end{aligned}
$$

The sign and absolute value of $\left(p_{+}+p_{-}\right) / 2$ are $\lambda_{p}$ and $\varepsilon_{p}$, respectively. Equation (27) like Eq. (17) defines the energy-shell. These variables were often used previously for quantization

on one of the two light fronts, $\tau_{+}=0$ or $\tau_{-}=0$ [40]. For the problem considered here, it is useful to keep the symmetry between $\tau_{+}$and $\tau_{-}$.

The projection operators $\left(\mathrm{I}_{4} \pm \check{\alpha}_{z}\right) / 2$ acquire a simple form and the interaction is diagonalized by introducing the light-fronts representation for the Dirac matrices,

$$
\begin{aligned}
& \gamma_{\text {light-fronts }}^{\mu}=\Lambda \gamma_{\text {Dirac }}^{\mu} \Lambda^{\dagger}, \\
& \Lambda \equiv \frac{1}{\sqrt{2}}\left(\begin{array}{cc}
\mathrm{I}_{2} & \hat{\sigma}_{z} \\
\mathrm{I}_{2} & -\hat{\sigma}_{z}
\end{array}\right), \\
& \Lambda \check{\alpha}_{z} \Lambda^{\dagger}=\left(\begin{array}{cc}
\mathrm{I}_{2} & 0_{2} \\
0_{2} & -\mathrm{I}_{2}
\end{array}\right) \text {, } \\
& \Lambda\left[\frac{1}{2}\left(\mathrm{I}_{4}+\check{\alpha}_{z}\right)\right] \Lambda^{\dagger}=\left(\begin{array}{cc}
\mathrm{I}_{2} & 0_{2} \\
0_{2} & 0_{2}
\end{array}\right), \\
& \Lambda\left[\frac{1}{2}\left(\mathrm{I}_{4}-\check{\alpha}_{z}\right)\right] \Lambda^{\dagger}=\left(\begin{array}{cc}
0_{2} & 0_{2} \\
0_{2} & \mathrm{I}_{2}
\end{array}\right) \text {, } \\
& \Lambda \check{\vec{\alpha}}_{\perp} \Lambda^{\dagger}=\left(\begin{array}{cc}
0_{2} & -\check{\vec{\omega}} \\
\check{\vec{\omega}} & 0_{2}
\end{array}\right) \text {, } \\
& \check{\vec{\omega}} \equiv\left(-\check{\sigma}_{y}, \check{\sigma}_{x}\right) \text {. }
\end{aligned}
$$


With this notation, the gauge-transformed two-center Dirac equation in the sharp ultrarelativistic limit in the light-fronts representation is

$$
\left(\begin{array}{c}
i \partial_{\tau_{+}}\left|G_{+}\right\rangle \\
i \partial_{\tau_{-}}\left|G_{-}\right\rangle
\end{array}\right)=\left(\begin{array}{cc}
\delta\left(\tau_{+}\right) B\left(\vec{r}_{\perp}\right) & \hat{h}_{0} \\
\hat{h}_{0}^{\dagger} & \delta\left(\tau_{-}\right) A\left(\vec{r}_{\perp}\right)
\end{array}\right)\left(\begin{array}{l}
\left|G_{+}\right\rangle \\
\left|G_{-}\right\rangle
\end{array}\right),
$$

where $\left|G_{+}\right\rangle$and $\left|G_{-}\right\rangle$are the upper and lower bi-spinor components of the Dirac wave function in the light-fronts representation

$$
\left(\begin{array}{c}
\left|G_{+}\right\rangle \\
\left|G_{-}\right\rangle
\end{array}\right) \equiv \Lambda|\Psi\rangle
$$

and

$$
\begin{aligned}
& \hat{h}_{0} \equiv \mathrm{I}_{2}-i \check{\vec{\omega}} \cdot \hat{\vec{p}}_{\perp}, \\
& A\left(\vec{r}_{\perp}, \vec{b}\right) \equiv Z_{A} \alpha \ln \left[\frac{\left(\vec{r}_{\perp}-\vec{b}\right)^{2}}{b^{2}}\right] \\
& B\left(\vec{r}_{\perp}, \vec{b}\right) \equiv Z_{B} \alpha \ln \left[\frac{\left(\vec{r}_{\perp}+\vec{b}\right)^{2}}{b^{2}}\right]
\end{aligned}
$$

The upper and lower bi-spinors are coupled by the free Hamiltonian. Each interacts directly with the external field of one ion and feels the field of the other ion through its coupling to the other bi-spinor.

Equation (35) has no discontinuities in the transverse direction. It is therefore useful to Fourier transform its solution with respect to $\vec{r}_{\perp}$. Two mixed bi-spinors wave-functions, $\left|g_{ \pm}\left(\vec{q}_{\perp} ; \tau_{+}, \tau_{-}\right)\right\rangle$, are then defined by

$$
\left|G_{ \pm}\left(\vec{r}_{\perp}, \tau_{+}, \tau_{-}\right)\right\rangle \equiv \int d \vec{q}_{\perp} e^{i \vec{r}_{\perp} \cdot \vec{q}_{\perp}}\left|g_{ \pm}\left(\vec{q}_{\perp} ; \tau_{+}, \tau_{-}\right)\right\rangle
$$

$\left|g_{+}\right\rangle$and $\left|g_{-}\right\rangle$, like $\left|G_{+}\right\rangle$and $\left|G_{-}\right\rangle$, are coupled by the free Hamiltonian.

\section{B. Free Dirac equation off the light fronts}

Off the light fronts, i.e. for $\tau_{+} \neq 0$ and $\tau_{-} \neq 0$, the wave function satisfies the free Dirac equation and Eq. (35) reduces to two coupled equations for the mixed bi-spinors 
$\left|g_{ \pm}\left(\vec{q}_{\perp} ; \tau_{+}, \tau_{-}\right)\right\rangle$

$$
\begin{aligned}
& i \frac{\partial}{\partial \tau_{+}}\left|g_{+}\right\rangle=\left(\mathrm{I}_{2}-i \check{\vec{\omega}} \cdot \vec{q}_{\perp}\right)\left|g_{-}\right\rangle, \\
& i \frac{\partial}{\partial \tau_{-}}\left|g_{-}\right\rangle=\left(\mathrm{I}_{2}+i \check{\vec{\omega}} \cdot \vec{q}_{\perp}\right)\left|g_{+}\right\rangle .
\end{aligned}
$$

As usual, the second-order equations decouple

$$
\frac{\partial^{2}}{\partial \tau_{+} \partial \tau_{-}}\left|g_{ \pm}\right\rangle=-\left(1+q_{\perp}^{2}\right)\left|g_{ \pm}\right\rangle
$$

where use was made of

$$
\left(\mathrm{I}_{2}-i \check{\vec{\omega}} \cdot \vec{q}_{\perp}\right)\left(\mathrm{I}_{2}+i \check{\vec{\omega}} \cdot \vec{q}_{\perp}\right)=\left(1+q_{\perp}^{2}\right) \mathrm{I}_{2}
$$

A solution to Eqs. (41, 42) is given, for example, by the plane waves of Eq. (18) which in the light-fronts representation are given by

$$
\begin{aligned}
& \left(\begin{array}{c}
\left|F_{+}^{p}\right\rangle \\
\left|F_{-}^{p}\right\rangle
\end{array}\right) \equiv \Lambda\left|\chi_{p}(\vec{r}, t)\right\rangle, \\
& \left|F_{ \pm}^{p}\right\rangle \equiv \int d \vec{q}_{\perp} e^{i \vec{r}_{\perp} \cdot \vec{q}_{\perp}}\left|f_{ \pm}^{p}\left(\vec{q}_{\perp} ; \tau_{+}, \tau_{-}\right)\right\rangle, \\
& \left|f_{ \pm}^{p}\left(\vec{q}_{\perp} ; \tau_{+}, \tau_{-}\right)\right\rangle=\delta\left(\vec{q}_{\perp}-\vec{p}_{\perp}\right) e^{-i\left(\tau_{-} p_{+}+\tau_{+} p_{-}\right)}\left|\Gamma_{ \pm}^{p}\right\rangle .
\end{aligned}
$$

The bi-spinors, $\left|\Gamma_{ \pm}^{p}\right\rangle$, (the upper and lower parts of $\Lambda\left|u_{p}\right\rangle$ ),

$$
\begin{aligned}
\left|\Gamma_{ \pm}^{p}\right\rangle= & \frac{(2 \pi)^{-3 / 2}}{2 \sqrt{\varepsilon_{p}\left(1+\varepsilon_{p}\right)}} \times \\
& {\left[\mathrm{I}_{2}\left(1+(-1)^{\lambda_{p}} p_{ \pm}\right) \pm i \check{\vec{\omega}} \cdot \vec{p}_{\perp}\right]\left( \pm \check{\sigma}_{z}\right)^{\lambda_{p}}\left|s_{p}\right\rangle }
\end{aligned}
$$

satisfy the simple relation

$$
\left|\Gamma_{-}^{p}\right\rangle=\frac{\mathrm{I}_{2}-i \check{\vec{\omega}} \cdot \vec{p}_{\perp}}{p_{+}}\left|\Gamma_{+}^{p}\right\rangle .
$$

These plane waves solve Eq. (35) off the light fronts in the limits $t \rightarrow \pm \infty$. They do not solve it for finite $t$, when $\vec{p}_{\perp}$ is no longer a good quantum number, as the singular interaction with the ions makes the wave function discontinuous at the light fronts. 


\section{The discontinuity across the light fronts}

It is standard procedure in wave-mechanics to form piece-wise solutions by satisfying continuity relations at the boundaries between free regions. It was shown in Refs. 32, 34 that a $\delta$-function singular interaction at a light front results in a discontinuity in the electron wave function which is given by a space-dependent phase shift. The proof is reviewed in appendix G, where it is shown that for our case of Eq. (35), the discontinuity is

$$
\begin{aligned}
& \left|G_{+}\left(\tau_{+}=0^{+}\right)\right\rangle=e^{-i B\left(\vec{r}_{\perp}, \vec{b}\right)}\left|G_{+}\left(\tau_{+}=0^{-}\right)\right\rangle, \\
& \left|G_{-}\left(\tau_{-}=0^{+}\right)\right\rangle=e^{-i A\left(\vec{r}_{\perp}, \vec{b}\right)}\left|G_{-}\left(\tau_{-}=0^{-}\right)\right\rangle .
\end{aligned}
$$

These phase shifts are derived from Eq. (35) in general for any $A\left(\vec{r}_{\perp}, \vec{b}\right)$ and $B\left(\vec{r}_{\perp}, \vec{b}\right)$, i.e. any functional dependence on the perpendicular coordinate. Here, $A\left(\vec{r}_{\perp}, \vec{b}\right)$ and $B\left(\vec{r}_{\perp}, \vec{b}\right)$ are given by Eqs. (38, 39).

Due to this space dependent phase-shift, the transverse momentum is not conserved and the Fourier components of Eq. (40) are mixed when the singularities at the light fronts are crossed,

$$
\begin{aligned}
\left|g_{+}\left(\vec{q}_{\perp} ; \tau_{+}=0^{+}\right)\right\rangle= & \int d \vec{p}_{\perp} Q_{Z_{B}}\left(\vec{p}_{\perp}-\vec{q}_{\perp},-\vec{b}\right) \\
& \times\left|g_{+}\left(\vec{p}_{\perp} ; \tau_{+}=0^{-}\right)\right\rangle, \\
\left|g_{-}\left(\vec{q}_{\perp} ; \tau_{-}=0^{+}\right)\right\rangle= & \int d \vec{p}_{\perp} Q_{Z_{A}}\left(\vec{p}_{\perp}-\vec{q}_{\perp}, \vec{b}\right) \\
& \times\left|g_{-}\left(\vec{p}_{\perp} ; \tau_{-}=0^{-}\right)\right\rangle,
\end{aligned}
$$

where

$$
Q_{Z}(\vec{\kappa}, \vec{b}) \equiv \frac{1}{(2 \pi)^{2}} \int d \vec{r}_{\perp} e^{i \overrightarrow{r_{\perp} \cdot \vec{\kappa}}}\left[\frac{\left(\vec{r}_{\perp}-\vec{b}\right)^{2}}{b^{2}}\right]^{-i \alpha Z} .
$$

Note that here $\vec{\kappa}$ and $\vec{b}$ are two-dimensional vectors in the $(x, y)$ plane. The continuity is recovered in the limit $Z \rightarrow 0$, as $Q_{Z}(\vec{\kappa}, \vec{b}) \rightarrow \delta(\vec{\kappa})$. The distribution $Q_{Z}(\vec{\kappa}, \vec{b})$ in general diverges. This divergence is an artifact of applying the sharp limit for the gauge-transformed interaction, Eqs. (11) and (12), for large $r_{\perp}$, i.e. outside its range of validity. The properties of the distribution $Q_{Z}(\vec{\kappa}, \vec{b})$ for finite charge are considered in appendix $\mathrm{D}$. 


\section{A PIECE-WISE SOLUTION TO THE SHARP DIRAC EQUATION}

In this section, the formalism that was introduced in section IV is used to obtain the transition amplitudes between asymptotic plane waves, $A_{k}^{(j)}$, defined in section III.

The singular interaction on the planes perpendicular to the trajectories of the ions, cut space-time along the light fronts into four regions, as is shown in Fig. 5. A piece-wise

solution is defined off the light fronts by $\left|g_{ \pm}\left(\vec{q}_{\perp} ; \tau_{+}, \tau_{-}\right)\right\rangle=\left|g_{ \pm}^{(\mathrm{i})}\left(\vec{q}_{\perp} ; \tau_{+}, \tau_{-}\right)\right\rangle$, where (i)= I for $\tau_{+}<0$ and $\tau_{-}<0,(\mathrm{i})=$ II for $\tau_{+}>0$ and $\tau_{-}<0$, (i)= III for $\tau_{+}<0$ and $\tau_{-}>0$, and (i) $=$ IV for $\tau_{+}>0$ and $\tau_{-}>0$. In each region, the wave function is continuous and solves the local free Dirac equation. At any time, except for $t \rightarrow \pm \infty$, the wave function extends in space through three (or two, at $t=0$ ) of these regions. The solution presented here is not complete in the sense that it does not include the solution on the light fronts; $\tau_{+}=0$ and $\tau_{-}=0$ are excluded. The physics on the light fronts may contribute to bound-free pair production. Thus, our present work is limited to free pair production.

\section{A. Initial condition and intermediate states}

Consider the initial condition, Eq. (22), of a single plane wave with the quantum numbers $j=\left\{\vec{j}, \lambda_{j}, s_{j}\right\}$, or, using light-front variables, $j=\left\{\vec{j}_{\perp}, j_{+}, j_{-}, s_{j}\right\}$, with the constraint $j_{+} j_{-}=$ $1+j_{\perp}^{2}$. The continuity off the light fronts gives the solution in region I,

$$
\left|g_{ \pm}^{\mathrm{I}}\left(\vec{q}_{\perp}\right)\right\rangle=\delta\left(\vec{j}_{\perp}-\vec{q}_{\perp}\right) e^{-i\left(\tau_{-} j_{+}+\tau_{+} j_{-}\right)}\left|\Gamma_{ \pm}^{j}\right\rangle
$$

where the bi-spinors $\left|\Gamma_{ \pm}^{j}\right\rangle$ are defined as in Eq. (48).

The solution in regions II and III is obtained by first applying Eq. (52) for the discontinuity across $\tau_{+}=0$ and Eq. (53) for the discontinuity across $\tau_{-}=0$ and then solving the coupled equations (41, 42) inside each of the intermediate space-time regions. We obtain in region II

$$
\left|g_{+}^{\mathrm{II}}\left(\vec{q}_{\perp}\right)\right\rangle=\exp \left[-i \tau_{-} j_{+}-i \tau_{+}\left(\frac{1+q_{\perp}^{2}}{j_{+}}\right)\right]
$$




$$
\begin{aligned}
& \times Q_{Z_{B}}\left(\vec{j}_{\perp}-\vec{q}_{\perp},-\vec{b}\right)\left|\Gamma_{+}^{j}\right\rangle, \\
\left|g_{-}^{\mathrm{II}}\left(\vec{q}_{\perp}\right)\right\rangle= & \left(\frac{I_{2}+i \overrightarrow{\vec{\omega}} \cdot \vec{q}_{\perp}}{j_{+}}\right)\left|g_{+}^{\mathrm{II}}\left(\vec{q}_{\perp}\right)\right\rangle,
\end{aligned}
$$

and in region III,

$$
\begin{aligned}
\left|g_{-}^{\mathrm{III}}\left(\vec{p}_{\perp}\right)\right\rangle= & \exp \left[-i \tau_{+} j_{-}-i \tau_{-}\left(\frac{1+p_{\perp}^{2}}{j_{-}}\right)\right] \\
& \times Q_{Z_{A}}\left(\vec{j}_{\perp}-\vec{p}_{\perp}, \vec{b}\right)\left|\Gamma_{-}^{j}\right\rangle, \\
\left|g_{+}^{\mathrm{III}}\left(\vec{p}_{\perp}\right)\right\rangle= & \left(\frac{I_{2}-i \overrightarrow{\vec{\omega}} \cdot \vec{p}_{\perp}}{j_{-}}\right)\left|g_{-}^{\mathrm{III}}\left(\vec{q}_{\perp}\right)\right\rangle .
\end{aligned}
$$

It is now apparent why the Fourier transform with respect to $\vec{r}_{\perp}$ and the definition of $\left|g_{ \pm}\left(\vec{q}_{\perp} ; \tau_{+}, \tau_{-}\right)\right\rangle$in Eq. (40) were needed. The simple discontinuity condition (50) at $\tau_{+}=0$ applies only to $\left|G_{+}\right\rangle$. The other bi-spinor $\left|G_{-}\right\rangle$is influenced indirectly by the field at $\tau_{+}=0$ through its coupling to $\left|G_{+}\right\rangle$. Likewise, at $\tau_{-}=0$ the simple discontinuity condition (51) for $\left|G_{-}\right\rangle$induces a non-trivial change in $\left|G_{+}\right\rangle$. The coupling between $\left|G_{+}\right\rangle$and $\left|G_{-}\right\rangle$in free space on either sides of the singular interaction is best described by Eqs. (41, 42) for their Fourier components with respect to $\vec{r}_{\perp}$. Thus, while the discontinuity conditions (52,53) for $\left|g_{ \pm}\right\rangle$seem more complicated than the discontinuity conditions (50,51) for $|G \pm\rangle$, using $\left|g_{ \pm}\right\rangle$ allows for a simple derivation of the complete spinor wave function in regions II and III.

It is a well known fact that two ions are needed in order to create an electron-positron pair. This can also be seen here. In the presence of ion B alone, for example, Eqs. (56) give the solution for $\tau_{+}>0$, including the asymptotic solution at $t_{f} \rightarrow \infty$. Projection on a plane wave then gives a conservation law for the positive light-front momentum. Likewise, in the presence of ion A alone the negative light front momentum is conserved.

$$
\begin{aligned}
& A_{k}^{(j)}\left(Z_{A}=0\right) \propto \delta\left(k_{+}-j_{+}\right), \\
& A_{k}^{(j)}\left(Z_{B}=0\right) \propto \delta\left(k_{-}-j_{-}\right) .
\end{aligned}
$$

A direct result from $k_{+}=j_{+}$or $k_{-}=j_{-}$is that the sign of the energy of the electron is the same before and after the collision. Thus, our formalism satisfies the known result that the passage of a uniformly moving charge does not induce a transition changing the sign of the 
energy. The presence of both ions defines a new region of space-time, region IV $\left(\tau_{ \pm}>0\right)$ which is the space between the ions $(-t<z<t)$ after the collision $(t>0)$, i.e. when the ions are already moving apart. It is shown below that the sign of the energy can change and pairs may be created in transitions from the initial state in region I $\left(t_{i} \rightarrow-\infty\right)$ to the final state in region IV $\left(t_{f} \rightarrow \infty\right)$.

The solution of the free Dirac equation in region IV is complicated by the non-trivial boundary conditions on the light fronts. Applying Eq. (52) again for the discontinuity across $\tau_{+}$and Eq. (53) for the discontinuity across $\tau_{-}$, we cross from regions II and III into region IV to obtain on the hyper-surfaces adjacent to the light fronts,

$$
\begin{aligned}
\left|g_{-}^{\mathrm{IV}}\left(\vec{k}_{\perp} ; \tau_{-}=0^{+}\right)\right\rangle= & \int d \vec{q}_{\perp} \exp \left[-i \tau_{+}\left(\frac{1+q_{\perp}^{2}}{j_{+}}\right)\right] \\
& \times Q_{Z_{A}}\left(\vec{q}_{\perp}-\vec{k}_{\perp}, \vec{b}\right) \\
& \times Q_{Z_{B}}\left(\vec{j}_{\perp}-\vec{q}_{\perp},-\vec{b}\right) \\
& \times\left(\frac{I_{2}+i \check{\vec{\omega}} \cdot \vec{q}_{\perp}}{j_{+}}\right)\left|\Gamma_{+}^{j}\right\rangle, \\
\left|g_{+}^{\mathrm{IV}}\left(\vec{k}_{\perp} ; \tau_{+}=0^{+}\right)\right\rangle= & \int d \vec{p}_{\perp} \exp \left[-i \tau_{-}\left(\frac{1+p_{\perp}^{2}}{j_{-}}\right)\right] \\
& \times Q_{Z_{B}}\left(\vec{p}_{\perp}-\vec{k}_{\perp}-\vec{b}\right) \\
& \times Q_{Z_{A}}\left(\vec{j}_{\perp}-\vec{p}_{\perp}, \vec{b}\right) \\
& \times\left(\frac{I_{2}-i \check{\vec{\omega}} \cdot \vec{p}_{\perp}}{j_{-}}\right)\left|\Gamma_{-}^{j}\right\rangle .
\end{aligned}
$$

Instead of solving now for $\left|g_{ \pm}^{\mathrm{IV}}\right\rangle$ at any $\tau_{ \pm}>0$, the transition amplitudes are obtained in the next subsection by defining the transition current and by applying Gauss' theorem for this current.

\section{B. The amplitudes}

The transition amplitudes $A_{k}^{(j)}$ were defined in Eq. (23),

$$
A_{k}^{(j)} \equiv \lim _{t_{f} \rightarrow \infty} \int d \vec{r} \chi_{k}^{\dagger}\left(\vec{r}, t_{f}\right) \psi^{(j)}\left(\vec{r}, t_{f}\right)
$$


The integrand is a component of a 4-vector current density, which is a conserved quantity (see appendix E). This transition current [35], $\left(\vec{J}^{(k, j)}, J_{0}^{(k, j)}\right)$, is defined by

$$
\begin{aligned}
& J_{0}^{(k, j)} \equiv \chi_{k}^{\dagger} \psi^{(j)} \\
& \vec{J}^{(k, j)} \equiv \chi_{k}^{\dagger} \check{\vec{\alpha}} \psi^{(j)} .
\end{aligned}
$$

An equivalent form for the transition current in terms of light-fronts representation wavefunctions includes

$$
J_{ \pm}^{(k, j)} \equiv J_{0}^{(k, j)} \pm J_{z}^{(k, j)}=2 F_{ \pm}^{k \dagger} G_{ \pm}^{(j)}
$$

It is now possible to use Gauss' theorem on the hyper-surface of the inner border of region IV to show that (see appendix $\mathrm{E}$ )

$$
\begin{aligned}
A_{k}^{(j)} \equiv & \lim _{t_{f} \rightarrow \infty} \int d \vec{r}_{\perp} \int_{-\infty}^{\infty} d z J_{0}^{(k, j)}\left(\vec{r}, t_{f}\right) \\
= & 2 \int d \vec{r}_{\perp} \int_{0^{+}}^{\infty} d \tau_{-} J_{+}^{(k, j)}\left(\vec{r}_{\perp}, \tau_{+}=0^{+}, \tau_{-}\right) \\
& -2 \int d \vec{r}_{\perp} \int_{0^{+}}^{\infty} d \tau_{+} J_{-}^{(k, j)}\left(\vec{r}_{\perp}, \tau_{+}, \tau_{-}=0^{+}\right) .
\end{aligned}
$$

The transition currents $J_{ \pm}^{(k, j)}$ are calculated from the results of the last subsection by using Eqs. (40,45,47,64).

$$
\begin{aligned}
J_{ \pm}^{(k, j)}\left(\vec{r}_{\perp}, \tau_{+}, \tau_{-}\right)= & 2 \int d \vec{p}_{\perp} \int d \vec{l}_{\perp} \exp \left[i \vec{r}_{\perp} \cdot\left(\vec{l}_{\perp}-\vec{p}_{\perp}\right)\right] \\
& \times\left\langle f_{ \pm}^{k}\left(\vec{p}_{\perp} ; \tau_{+}, \tau_{-}\right) \mid g_{ \pm}^{\mathrm{IV}}\left(\vec{l}_{\perp} ; \tau_{+}, \tau_{-}\right)\right\rangle
\end{aligned}
$$

Integrating over $\vec{r}_{\perp}$ and using the explicit expression (47) for the plane waves,

$$
\begin{aligned}
A_{k}^{(j)}= & \int_{0^{+}}^{\infty} d \tau_{-} e^{i \tau_{-} k_{+}}\left\langle\Gamma_{+}^{k} \mid g_{+}^{\mathrm{IV}}\left(\vec{k}_{\perp} ; \tau_{+}=0^{+}, \tau_{-}\right)\right\rangle \\
& -2 \int_{0^{+}}^{\infty} d \tau_{+} e^{i \tau_{+} k_{-}}\left\langle\Gamma_{-}^{k} \mid g_{-}^{\mathrm{IV}}\left(\vec{k}_{\perp} ; \tau_{+}, \tau_{-}=0^{+}\right)\right\rangle .
\end{aligned}
$$

The amplitudes are finally obtained by substituting Eqs. (60,61) and integrating over $\tau_{ \pm}$. The integration over $\tau_{ \pm}$would have given a $\delta$-function conservation law for the light-front momenta, had it been on the complete line $-\infty<\tau_{ \pm}<\infty$. Instead, the integrals on the 
half lines $0<\tau_{ \pm}<\infty$ are regulated in the usual way with an infinitesimal small constant, $\eta$ 41].

$$
\int_{0^{+}}^{\infty} d \tau \exp (i \tau \kappa)=\frac{-i}{\kappa+i \eta}
$$

The transition amplitudes corresponding to the exact solution of the sharp Dirac equation off the light fronts are

$$
\begin{aligned}
A_{k}^{(j)}= & \frac{(2 \pi)^{3}}{i \pi} \times \\
& \left\{\int d \vec{p}_{\perp} Q_{Z_{B}}\left(\vec{p}_{\perp}-\vec{k}_{\perp},-\vec{b}\right) Q_{Z_{A}}\left(\vec{j}_{\perp}-\vec{p}_{\perp}, \vec{b}\right)\right. \\
& \times \frac{\left\langle\Gamma_{+}^{k}\left|I_{2}-i \check{\vec{\omega}} \cdot \vec{p}_{\perp}\right| \Gamma_{-}^{j}\right\rangle}{j_{-} k_{+}-\left(1+p_{\perp}^{2}\right)+i \eta(-1)^{\lambda_{j}}} \\
& -\int d \vec{q}_{\perp} Q_{Z_{A}}\left(\vec{q}_{\perp}-\vec{k}_{\perp}, \vec{b}\right) Q_{Z_{B}}\left(\vec{j}_{\perp}-\vec{q}_{\perp},-\vec{b}\right) \\
& \left.\times \frac{\left\langle\Gamma_{-}^{k}\left|I_{2}+i \check{\vec{\omega}} \cdot \vec{q}_{\perp}\right| \Gamma_{+}^{j}\right\rangle}{j_{+} k_{-}-\left(1+q_{\perp}^{2}\right)+i \eta(-1)^{\lambda_{j}}}\right\},
\end{aligned}
$$

where the infinitesimal small, positive constant, $\eta$, can be omitted for pair-production amplitudes corresponding to $E_{j}<0$ and $E_{k}>0$, i.e. $j_{ \pm} k_{\mp}<0$. This is an interesting result. In the ultra-relativistic limit of Eq. (5), the asymptotic time evolution of the gauge-transformed electron wave function, $|\Psi(\vec{r}, t)\rangle$, is exactly given by these amplitudes. An exponential, nonperturbative dependence on the coupling constant $\alpha Z$ appears here as non-trivial phases

in the integral representation of the distributions $Q_{Z_{A}}\left(\vec{\kappa}_{\perp}, \vec{b}\right)$ and $Q_{Z_{B}}\left(\vec{\kappa}_{\perp},-\vec{b}\right)$ which were defined in Eq. (54). The two terms in Eq. (69) correspond to two different time-orderings of the interaction with the ions. In the next subsection, they are shown to reduce in the smallcoupling perturbative limit to the well known two photon exchange diagrams as depicted in Fig. 7. For vanishing charges, as could be expected,

$$
A_{k}^{(j)}\left(Z_{A}=0, Z_{B}=0\right)=\delta(\vec{k}-\vec{j}) \delta_{\lambda_{k}, \lambda_{j}} \delta_{s_{k}, s_{j}} .
$$

\section{The perturbative limit}

The small-charge perturbative-limit of the pair-production amplitude was calculated in Ref. [18. To leading order in $\alpha Z$ (second order), the amplitude is given by a sum over two 
diagrams, where each diagram describes a two-photon exchange process. The second-order perturbation-theory result, $\mathcal{S}_{k}^{2}{ }^{(j)}$, for the transition amplitude between an initial negativeenergy state $j=\left\{\vec{j}, \lambda_{j}=1, s_{j}\right\}$ and a final positive-energy state $k=\left\{\vec{k}, \lambda_{k}=0, s_{k}\right\}$, is given by Eqs. (24-32) of Ref. [18]. In the ultra-relativistic limit, $\beta \rightarrow 1$ and large $\gamma$, the perturbative result reduces to

$$
\begin{aligned}
\mathcal{S}_{k}^{2(j)}= & \int d \vec{p}_{\perp} \exp \left[-i \vec{b} \cdot\left(2 \vec{p}_{\perp}-\vec{j}_{\perp}-\vec{k}_{\perp}\right)\right] \\
& \times \frac{i 8\left(\alpha Z_{A}\right)\left(\alpha Z_{B}\right)}{\left(\vec{p}_{\perp}-\vec{k}_{\perp}\right)^{2}\left(\vec{p}_{\perp}-\vec{j}_{\perp}\right)^{2}} \\
& \times \frac{\left\langle\Gamma_{+}^{k}\left|I_{2}-i \check{\vec{\omega}} \cdot \vec{p}_{\perp}\right| \Gamma_{-}^{j}\right\rangle}{j_{-} k_{+}-\left(1+p_{\perp}^{2}\right)} \\
& -\int d \vec{q}_{\perp} \exp \left[i \vec{b} \cdot\left(2 \vec{q}_{\perp}-\vec{j}_{\perp}-\vec{k}_{\perp}\right)\right] \\
& \times \frac{i 8\left(\alpha Z_{A}\right)\left(\alpha Z_{B}\right)}{\left(\vec{q}_{\perp}-\vec{k}_{\perp}\right)^{2}\left(\vec{q}_{\perp}-\vec{j}_{\perp}\right)^{2}} \\
& \times \frac{\left\langle\Gamma_{-}^{k}\left|I_{2}+i \breve{\vec{\omega}} \cdot \vec{q}_{\perp}\right| \Gamma_{+}^{j}\right\rangle}{j_{+} k_{-}-\left(1+q_{\perp}^{2}\right)} .
\end{aligned}
$$

A transformation to the light-fronts representation was used here to obtain bi-spinor bra-kets from the 4-spinor bra-kets of Ref. [18]. For example, using $\Lambda^{\dagger} \Lambda \equiv \mathrm{I}_{4}$,

$$
\begin{aligned}
& \left\langle u_{k}\left|\left(I_{4}-\check{\alpha}_{z}\right)\left(\check{\alpha} \cdot \vec{p}_{\perp}+\check{\gamma}^{0}\right)\left(I_{4}+\check{\alpha}_{z}\right)\right| u_{j}\right\rangle \\
& \equiv\left\langle\Gamma_{+}^{k}\left|I_{2}-i \check{\vec{\omega}} \cdot \vec{p}_{\perp}\right| \Gamma_{-}^{j}\right\rangle .
\end{aligned}
$$

It is interesting to compare the perturbative result of Eq. (71) to our non-perturbative result of Eq. (69). In the small-charge limit of $\alpha Z \rightarrow 0$, after proper regularization, the leading-order perturbative limit for $Q_{Z}$ from appendix $\square$ can be used,

$$
Q_{Z}(\vec{\kappa}, \vec{b}) \rightarrow \delta(\vec{\kappa})-\frac{i \alpha Z}{\pi} \frac{1}{\kappa^{2}} \exp [i \vec{b} \cdot \vec{\kappa}]
$$

Direct substitution shows that in this limit the non-perturbative result of Eq. (69) exactly reproduces the perturbative result of Eq. (71). 


\section{CONCLUSIONS AND OUTLOOK}

We have used a gauge transformation to obtain a useful ultra-relativistic limit for the twocenter Dirac equation, which allows for an exact solution off the planes perpendicular to the ions' trajectories, i.e. off the light fronts. In general, the amplitudes of the gauge-transformed Dirac equation are related to the amplitudes of the original equation in a non-trivial way due to long-range Coulomb effects. For symmetric collisions, and calculations in the collider frame, some of these long-range effects cancel. The two different amplitudes are then related by a series expansion, and, to leading order, they are equal.

The amplitudes were calculated here in the ultra-relativistic limit, assuming $\gamma$ to be large. No assumption was made on the value of the charge times the fine-structure constant $Z \alpha$. When taking the limit of small $Z \alpha$, we are able to show a complete agreement with the ultra-relativistic limit of the expression obtained from standard second-order perturbation theory [18]. In second-order perturbation theory, pair production is described as a two photon exchange process in which each ion exchanges one photon with a negative-energy electron. The negative energy electron is kicked off its energy shell by the first interaction and then kicked back to the energy shell by the second ion, but with a positive energy. The two diagrams that contribute to the amplitude differ in the time order of these photon exchanges, or 'kicks'. Our result, which provides a very similar physical picture of pair production as a 'two-kicks' process, is obtained in the ultra-relativistic limit within a rather different, and completely non-perturbative approach.

In our work, the electromagnetic fields of the ions are confined to the light fronts by the extreme Lorentz contraction and by a choice of a particular gauge designed to remove the long-range Coulomb effects. In this gauge, as the velocity of the ions approaches the velocity of light, each ion carries with it, perpendicular to its trajectory, a wall of singular electromagnetic interaction. An initial plane wave in the space between the approaching ions acquires a space-dependent phase shift as it is swept by this singular-interaction wall. A single plane wave between the ions gives a distribution of local plane waves in the space 
behind each ion. Had there been only one ion, no transition would be allowed between the negative-energy continuum and the positive-energy continuum, i.e. no pairs could be produced. Pairs are produced because, as the ions move past each other, the two phaseshift planes collide. After the peripheral collision, as the ions move apart, the solution in the space between them is determined by the non-trivial boundary conditions at the light fronts. The main result of this work, the exact integral representation for the free pair creation amplitudes of Eq. (69), is finally obtained in this framework by calculating the transition currents flowing from the light fronts into the space between the separating ions. The two terms correspond to the two time orderings of the interaction of the two phase-shift walls with the electronic wave function. In the perturbative limit of a small coupling constant, the effect of the singular field perpendicular to each ion reduces to a single photon exchange. For finite charges of the ions, the perturbative linear dependence of the amplitudes on each charge is replaced by non-perturbative, nontrivial phases in our integral representation.

Numerical evaluation of the non-perturbative effects, differential cross sections, and applications to multiple pair production will be considered in future work.

\section{ACKNOWLEDGMENTS}

The authors are happy to acknowledge helpful conversations with Prof. J. Macek and Prof. M. R. Strayer. This work was supported by the National Science Foundation through a grant for the Institute for Theoretical Atomic and Molecular Physics at Harvard University and Smithsonian Astrophysical Observatory.

\section{APPENDIX A: THE SHARP, ULTRA-RELATIVISTIC LIMIT}

In this appendix, we will outline the derivation of the $\delta$-function limit of the electromagnetic interactions, $W_{A}$ and $W_{B}$, given in (11) and (12) beginning with their definitions given in (9) and (10), respectively. (The same limit has been previously obtained; see Refs. (32,34,39). 
In the limit of extreme ultra-relativistic collisions, one may neglect terms in the interaction proportional to $\gamma^{-2}$. It is then possible to first set $\beta \rightarrow 1$ and then use

$$
\begin{aligned}
\eta(\tau ; a, b) & \equiv\left(\frac{b^{2}}{\gamma^{2}}+\tau^{2}\right)^{-1 / 2}-\left(\frac{a^{2}}{\gamma^{2}}+\tau^{2}\right)^{-1 / 2} \\
& \stackrel{\gamma \gg a_{a} b}{\longrightarrow} \delta(\tau) \ln \left(\frac{a^{2}}{b^{2}}\right)
\end{aligned}
$$

It is easy to verify that in the limit $\gamma \gg a, b$,

$$
\int_{-\infty}^{\infty} d \tau \eta(\tau ; a, b) \rightarrow \ln \left(\frac{a^{2}}{b^{2}}\right),
$$

and that in the same limit

$$
\eta(\tau \neq 0 ; a, b) \propto \frac{a^{2}-b^{2}}{\gamma^{2}} \rightarrow 0 .
$$

\section{APPENDIX B: THE GAUGE TRANSFORMED AMPLITUDES}

In section III, the transition amplitudes $S_{k}^{(j)}$ and $A_{k}^{(j)}$ were defined and an exact relation, Eq. (24), was established between them. However, this relation is not always useful as it involves infinite integrals and sums over the complete plane-wave basis set. In this appendix, a series expansion in inverse powers of the asymptotic time will be shown to relate $S_{k}^{(j)}$ and $A_{k}^{(j)}$ in a simpler way for symmetric collisions and calculations in the collider frame.

Two evolution operators can be defined for $|\Phi\rangle$ and $|\Psi\rangle$, from Eq. (1) and (8), respectively,

$$
\begin{aligned}
& \left|\Phi\left(\vec{r}, t_{f}\right)\right\rangle=\hat{\mathcal{V}}\left(t_{f}, t_{i}\right)\left|\Phi\left(\vec{r}, t_{i}\right)\right\rangle \\
& \left|\Psi\left(\vec{r}, t_{f}\right)\right\rangle=\hat{\mathcal{U}}\left(t_{f}, t_{i}\right)\left|\Psi\left(\vec{r}, t_{i}\right)\right\rangle .
\end{aligned}
$$

They are related by the gauge transformation of Eq. (7)

$$
\hat{\mathcal{U}}\left(t_{f}, t_{i}\right)=U\left(z, t_{f}\right) \hat{\mathcal{V}}\left(t_{f}, t_{i}\right) U^{\dagger}\left(z, t_{i}\right) .
$$

The amplitudes are given by 


$$
\begin{aligned}
& S_{k}^{(j)}=\lim _{\substack{t_{i} \rightarrow-\infty \\
t_{f} \rightarrow \infty}}\left\langle\chi_{k}\left(\vec{r}, t_{f}\right)\left|\hat{\mathcal{V}}\left(t_{f}, t_{i}\right)\right| \chi_{j}\left(\vec{r}, t_{i}\right)\right\rangle, \\
& A_{k}^{(j)}=\lim _{\substack{t_{i} \rightarrow-\infty \\
t_{f} \rightarrow \infty}}\left\langle\chi_{k}\left(\vec{r}, t_{f}\right)\left|\hat{\mathcal{U}}\left(t_{f}, t_{i}\right)\right| \chi_{j}\left(\vec{r}, t_{i}\right)\right\rangle .
\end{aligned}
$$

A direct substitution gives

$$
S_{k}^{(j)}=\lim _{\substack{t_{i} \rightarrow-\infty \\ t_{f} \rightarrow \infty}}\left\langle\chi_{k}\left(\vec{r}, t_{f}\right)\left|U^{\dagger}\left(z, t_{f}\right) \hat{\mathcal{U}}\left(t_{f}, t_{i}\right) U\left(z, t_{i}\right)\right| \chi_{j}\left(\vec{r}, t_{i}\right)\right\rangle .
$$

The asymptotic expressions,

$$
\begin{gathered}
U\left(z, t_{i}\right) \stackrel{t_{i} \rightarrow-\infty}{\longrightarrow} \frac{\left[2 \gamma\left(\left|t_{i}\right|+z\right) / b\right]^{i Z_{A} \alpha}}{\left[2 \gamma\left(\left|t_{i}\right|-z\right) / b\right]^{i Z_{B} \alpha}} \\
U^{\dagger}\left(z, t_{f}\right) \stackrel{t_{f} \rightarrow \infty}{\longrightarrow} \frac{\left[2 \gamma\left(t_{f}-z\right) / b\right]^{i Z_{A} \alpha}}{\left[2 \gamma\left(t_{f}+z\right) / b\right]^{i Z_{B} \alpha}}
\end{gathered}
$$

reduce, for symmetric collisions, $\left(Z \equiv Z_{A}=Z_{B}\right)$, to power series in $z / t$

$$
\begin{aligned}
& U\left(z, t_{i}\right) \approx 1+i 2 Z \alpha \frac{z}{\left|t_{i}\right|}+\ldots \\
& U^{\dagger}\left(z, t_{f}\right) \approx 1-i 2 Z \alpha \frac{z}{t_{f}}+\ldots
\end{aligned}
$$

Substituting these power series in Eq. (B6) and integrating term by term one gets,

$$
\begin{aligned}
S_{k}^{(j) \approx} & A_{k}^{(j)} \\
& +i 2 Z \alpha \lim _{\substack{t_{i} \rightarrow-\infty \\
t_{f} \rightarrow \infty}}\left\langle\chi_{k}\left(\vec{r}, t_{f}\right)\left|\hat{\mathcal{U}}\left(t_{f}, t_{i}\right) \frac{z}{\left|t_{i}\right|}\right| \chi_{j}\left(\vec{r}, t_{i}\right)\right\rangle \\
& -i 2 Z \alpha \lim _{\substack{t_{i} \rightarrow-\infty \\
t_{f} \rightarrow \infty}}\left\langle\chi_{k}\left(\vec{r}, t_{f}\right)\left|\frac{z}{t_{f}} \hat{\mathcal{U}}\left(t_{f}, t_{i}\right)\right| \chi_{j}\left(\vec{r}, t_{i}\right)\right\rangle+\cdots
\end{aligned}
$$

Using completeness, Eq. (B11) can equivalently be written as

$$
\begin{aligned}
S_{k}^{(j) \approx} & A_{k}^{(j)} \\
& +i 2 Z \alpha \sum_{l}\left[A_{k}^{(l)} \lim _{t_{i} \rightarrow-\infty}\left\langle\chi_{k}\left(\vec{r}, t_{f}\right)\left|\frac{z}{\left|t_{i}\right|}\right| \chi_{j}\left(\vec{r}, t_{i}\right)\right\rangle\right. \\
& \left.-A_{l}^{(j)} \lim _{t_{f} \rightarrow \infty}\left\langle\chi_{k}\left(\vec{r}, t_{f}\right)\left|\frac{z}{t_{f}}\right| \chi_{j}\left(\vec{r}, t_{i}\right)\right\rangle\right]+\cdots
\end{aligned}
$$

It is clear that $S_{k}^{(j)}$ and $A_{k}^{(j)}$ are in general different. For non-symmetric collisions, the relation between them involves, for example, a highly oscillatory, $z$-dependent phase which 
explicitly depends on $\gamma$. However, this phase cancels for the interesting case of symmetric collisions. The first-order corrections to $S_{k}^{(j)} \approx A_{k}^{(j)}$ decrease linearly with time and have a functional nature of polarization effects. Higher-order corrections are higher moments of $z / t$.

\section{APPENDIX C: THE DISCONTINUITY AT THE LIGHT FRONTS}

In this appendix, the discontinuities of the spinor wave function at the light fronts (at $\tau_{+}=0$ and at $\tau_{-}=0$, excluding only $\tau_{+}=\tau_{-}=0$ ) are deduced from Eq. (35). Previous derivations of the discontinuity of a wave function due to an ultra-relativistic charge are reviewed.

At one light front $\left(\tau_{+}=0, \tau_{-} \neq 0\right)$, Eq. (35) for $\left|G_{+}\right\rangle$reads,

$$
i \partial_{\tau_{+}}\left|G_{+}\right\rangle=\hat{h}_{0}\left|G_{-}\right\rangle+B\left(\vec{r}_{\perp}\right) \delta\left(\tau_{+}\right)\left|G_{+}\right\rangle
$$

The $\delta$-function singularity renders $\left|G_{+}\right\rangle$discontinuous at $\tau_{+}=0$, as can be seen by integrating both hand sides of Eq. (C1) with respect to $\tau_{+}$from $-\epsilon$ to $\epsilon$ and taking the limit $\epsilon \rightarrow 0$,

$$
\left|G_{+}\left(\tau_{+}=0^{+}\right)\right\rangle \neq\left|G_{+}\left(\tau_{+}=0^{-}\right)\right\rangle \text {. }
$$

An auxiliary bi-spinor can be defined by a piece-wise gauge transformation,

$$
\left|\tilde{G}_{+}\right\rangle \equiv \exp \left[i B\left(\vec{r}_{\perp}\right) \theta\left(\tau_{+}\right)\right]\left|G_{+}\right\rangle
$$

Direct substitution gives,

$$
i \partial_{\tau_{+}}\left|\tilde{G}_{+}\right\rangle=\exp \left[i B\left(\vec{r}_{\perp}\right) \theta\left(\tau_{+}\right)\right] \hat{h}_{0}\left|G_{-}\right\rangle
$$

The auxiliary bi-spinor is continuous at $\tau_{+}=0$, as can be seen by operating on both sides of Eq. (C4) with $\lim _{\epsilon \rightarrow 0} \int_{-\epsilon}^{\epsilon} d \tau_{+}$, obtaining

$$
\left|\tilde{G}_{+}\left(\tau_{+}=0^{+}\right)\right\rangle=\left|\tilde{G}_{+}\left(\tau_{+}=0^{-}\right)\right\rangle
$$


The continuity of $\left|\tilde{G}_{+}\right\rangle$at $\tau_{+}=0\left(\tau_{-} \neq 0\right)$, implies the discontinuity of Eq. (50) for $\left|G_{+}\right\rangle$. Likewise, a continuity of

$$
\left|\tilde{G}_{-}\right\rangle \equiv \exp \left[i A\left(\vec{r}_{\perp}\right) \theta\left(\tau_{-}\right)\right]\left|G_{-}\right\rangle
$$

at $\tau_{-}=0,\left(\tau_{+} \neq 0,\right)$ implies the discontinuity of Eq. (51) for $\left|G_{-}\right\rangle$.

This Heavyside step-function, space-dependent, phase discontinuity was previously obtained in Ref. [32]. In earlier work [34, a gauge transformation was used to establish the fact that the electromagnetic field of a charge which is moving at the speed of light can be equivalently given by gauge potentials with a $\delta$-function singularity at the light front, or by gauge potentials with only a step-function discontinuity there. The wave function of a particle interacting with this field is discontinuous or continuous, depending on the gauge choice. We choose to work with such a gauge that would give a sharp interaction and a discontinuous spinor wave function, yet we have used here other gauges to find the discontinuities in an explicit form.

\section{APPENDIX D: THE MOMENTUM-TRANSFER DISTRIBUTION}

When the singularities at the light fronts are crossed, the transverse momentum changes. The distribution for this momentum change is given in section IV by Eq. (54),

$$
Q_{Z}(\vec{\kappa}, \vec{b}) \equiv \frac{1}{(2 \pi)^{2}} \int d \vec{r}_{\perp} e^{i \vec{r}_{\perp} \cdot \vec{\kappa}}\left[\frac{\left(\vec{r}_{\perp}-\vec{b}\right)^{2}}{b^{2}}\right]^{-i \alpha Z}
$$

As mentioned in section IV, divergence of $Q_{Z}(\vec{\kappa}, \vec{b})$ is an artifact of the ultra-relativistic approximation used in Eq. (11) and (12). The integral over this distribution converges and is normalized to 1

$$
\int d \vec{\kappa} Q_{Z}(\vec{\kappa}, \vec{b})=1
$$

For a vanishing charge, $Q_{Z=0}(\vec{\kappa}, \vec{b})=\delta(\vec{\kappa})$, but for finite charge $Z \neq 0$, this distribution diverges both for vanishing and finite momentum transfer $\vec{\kappa}$. In this appendix, we show that 
for $\vec{\kappa} \neq 0$ in the perturbative limit, $\alpha Z \ll 1$, and after proper regularization, the leading order correction to the $\delta$-function is given by Eq. (73), i.e.

$$
Q_{Z}(\vec{\kappa}, \vec{b}) \rightarrow \delta(\vec{\kappa})-\frac{i \alpha Z}{\pi} \frac{1}{\kappa^{2}} \exp (i \vec{b} \cdot \vec{\kappa})
$$

Integrating first over the angular variable,

$$
Q_{Z}(\vec{\kappa} \neq 0, \vec{b})=\frac{b^{2} \exp (i \vec{b} \cdot \vec{\kappa})}{2 \pi} \int_{0}^{\infty} d s J_{0}[s b \kappa] s^{1-i 2 \alpha Z}
$$

where $b=|\vec{b}|, \kappa=|\vec{\kappa}|$, and $J_{0}$ is the Bessel function. The integral over $s$ diverges, but can be regulated for finite $\kappa$ in the limit of $\alpha Z \ll 1$.

Using, for example, Eq. (6.631.1) in Ref. [42] and Eq. (13.5.1) in Ref. [43] one has

$$
\begin{aligned}
& \lim _{\epsilon \rightarrow 0} \frac{1}{2 \pi} \int_{0}^{\infty} d s e^{-\epsilon s^{2}} J_{0}[s b \kappa] s^{1-i 2 \alpha Z} \\
& =\lim _{\epsilon \rightarrow 0} \frac{\Gamma(1-i \alpha Z)}{4 \pi \epsilon^{1-i \alpha Z}}{ }_{1} F_{1}\left(1-i \alpha Z, 1 ; \frac{-(b \kappa)^{2}}{4 \epsilon}\right) \\
& =\lim _{\epsilon \rightarrow 0} \frac{1}{4 \pi \epsilon} \exp \left[-(b \kappa)^{2} / 4 \epsilon\right]\left(\frac{i b \kappa}{2 \epsilon}\right)^{-i 2 \alpha Z} \\
& -\frac{i \alpha Z}{\pi}(b \kappa)^{-2} e^{-\pi \alpha Z} \frac{\Gamma(-i \alpha Z)}{\Gamma(+i \alpha Z)}\left(\frac{i b \kappa}{2}\right)^{+i 2 \alpha Z}
\end{aligned}
$$

where ${ }_{1} F_{1}$ is the confluent hypergeometric function. Eq. (73) is now obtained by taking $\alpha Z \rightarrow 0$ and using $\delta(b \vec{\kappa}) \equiv \lim _{\epsilon \rightarrow 0} \frac{1}{4 \pi \epsilon} \exp \left[-(b \kappa)^{2} / 4 \epsilon\right]$. Note that the limit $\epsilon \rightarrow 0$ can only be taken after taking the perturbative limit $\alpha Z \rightarrow 0$.

\section{APPENDIX E: THE TRANSITION CURRENT}

As we are unaware of an appropriate reference, we prove in this appendix that the transition 4-current density defined in Eq. (63) is conserved. In fact, any two solutions of the free Dirac equation can be used to define a conserved current in a similar way. This

proof is very similar to the one found in textbooks proving the probability current to be conserved [2].

Both $\chi_{k}$ and $\psi^{(j)}$ solve in region IV the free Dirac equation in the Dirac representation 


$$
\begin{array}{r}
i \frac{\partial}{\partial t} \psi^{(j)}(\vec{r}, t)=\left[-i \check{\alpha} \cdot \vec{\nabla}+\check{\gamma^{0}}\right] \psi^{(j)}(\vec{r}, t), \\
i \frac{\partial}{\partial t} \chi_{k}(\vec{r}, t)=\left[-i \check{\alpha} \cdot \vec{\nabla}+\check{\gamma^{0}}\right] \chi_{k}(\vec{r}, t) .
\end{array}
$$

Multiplying Eq. (E1) from the left by the adjoint of $\chi_{k}$ gives

$$
i \chi_{k}^{\dagger} \frac{\partial \psi^{(j)}}{\partial t}=-i \chi_{k}^{\dagger} \check{\alpha} \cdot \vec{\nabla} \psi^{(j)}+\chi_{k}^{\dagger} \check{\gamma}^{0} \psi^{(j)}
$$

Multiplying the Hermitian conjugate of Eq. (E2) from the right by $\psi^{(j)}$ gives

$$
-i \frac{\partial \chi_{k}^{\dagger}}{\partial t} \psi^{(j)}=i\left(\check{\alpha} \cdot \vec{\nabla} \chi_{k}^{\dagger}\right) \psi^{(j)}+\chi_{k}^{\dagger} \check{\gamma^{0}} \psi^{(j)}
$$

Subtracting Eq. (E4) from Eq. (E3) gives

$$
\frac{\partial}{\partial t}\left(\chi_{k}^{\dagger} \psi^{(j)}\right)=-\vec{\nabla} \cdot\left(\chi_{k}^{\dagger} \check{\alpha} \psi^{(j)}\right)
$$

where the Hermiticity of the Dirac matrices has been used. Using the definition of the transition current in Eq. (63), Eq. (E5) is reveled as the continuity equation

$$
\frac{\partial}{\partial t} J_{0}^{(j, k)}+\vec{\nabla} \cdot \vec{J}^{(j, k)}=0
$$

proving the transition-current density to be conserved.

\section{APPENDIX F: APPLICATION OF GAUSS' THEOREM}

As appendix $\mathrm{E}$ shows, the transition 4-current, defined in Eq. (63), is a conserved quantity,

$$
\frac{\partial J^{\mu}}{\partial x^{\mu}}=0
$$

Integrating Eq. ([F1) over any empty space-time hyper-volume, $V$, and applying Guass' theorem to convert the volume integral into a surface integral over the hyper-surface $S$ enclosing $V$, in general gives,

$$
\int_{S} d \sigma J^{\mu} n_{\mu}=0
$$


where the unit 4 -vector $n_{\mu}$ is defined as the outward pointing normal to $S$.

For our purposes, it is useful to apply Eq. (F2) to the space-time region IV, defined in Fig. 5 by $\tau_{ \pm}>0$. The closed hyper-surface $S$ enclosing region IV is made of the following open hyper-surfaces: (i) $t=t_{f} \rightarrow+\infty$, (ii) $\tau_{+}=0^{+}, \tau_{-}>0$, (iii) $\tau_{-}=0^{+}, \tau_{+}>0$, (iv) $x \rightarrow \pm \infty$, and (v) $y \rightarrow \pm \infty$; (see Fig. 6). Writing Eq. (F2) for this surface gives

$$
\begin{aligned}
0= & \lim _{t_{f} \rightarrow \infty} \int d r_{\perp} \int_{+\infty}^{-\infty} J_{0}\left(\vec{r}, t_{f}\right) \\
& -2 \int d r_{\perp} \int_{+\infty}^{0^{+}} d \tau_{-} J_{+}\left(\vec{r}_{\perp}, \tau_{+}=0^{+}, \tau_{-}\right) \\
& -2 \int d r_{\perp} \int_{0^{+}}^{+\infty} d \tau_{+} J_{-}\left(\vec{r}_{\perp}, \tau_{+}, \tau_{-}=0^{+}\right),
\end{aligned}
$$

where use was made of the fact that in any physical situation, i.e. for a square-integrable wavepacket, the currents vanish as $\vec{r} \rightarrow \infty$. The hyper-surfaces (iv) and (v) do not contribute to the integral of Eq. $(\mathbb{F 2})$. The factors of 2 arise from the Jacobian relating the original differentials to the differentials for the light-front variables, and the negative sign in the second and third terms arise because the unit normal vectors $\hat{n}_{ \pm}$are directed outside the volume $V$, i.e. $J \cdot \hat{n}_{ \pm}=-J_{ \pm}$. This completes our proof of Eq. (65). 


\section{REFERENCES}

[1] C. Itzykson and J.-B. Zuber, "Quantum Field Theory", (McGraw-Hill, New York, 1980).

[2] W. Greiner, B. Müller, and J. Rafelski, "Quantum Electrodynamics of strong fields", (SpringerVerlag, Berlin Heidelberg, 1985).

[3] J. Eichler and W.E. Meyerhof, "Relativistic Atomic Collisions", (Academic Press, San Diego, 1995).

[4] S.S. Gershtein and Y.B. Zeldovich, Sov. Phys. JETP 30, 358 (1970).

[5] M.S. Marinov and V.S. Popov, Sov. Phys. JETP 38, 1069 (1974); 41, 205 (1975).

[6] B. Müller, J. Rafelski, and W. Greiner, Phys. Lett. B47, 5 (1973).

[7] J. Rafelski and B. Müller, Phys. Lett. B65, 205 (1976).

[8] V.I. Lisin, M.S. Marinov and V.S. Popov, Phys. Lett. B69, 141 (1977).

[9] see, for example, J. Eichler, Phys. Rep. 193, 165 (1990), and references therein.

[10] C.R. Vane, S. Datz, P.F. Dittner, H.F. Krause, C. Bottcher, M.R. Strayer, R. Schuch, H. Gao and R. Hutton, Phys. Rev. Lett. 69, 1911 (1992).

[11] A. Belkacem, H. Gould, B. Feinberg, R. Bossingham and W.E. Meyerhof, Phys. Rev. Lett. 71, 1514 (1993).

[12] R. Baur et al., Phys. Lett. B 322, 471 (1994).

[13] A. Belkacem, H. Gould, B. Feinberg, R. Bossingham and W.E. Meyerhof, Phys. Rev. Lett. 73 2432 (1994).

[14] C.R. Vane, S. Datz, P.F. Dittner, H.F. Krause, R. Schuch, H. Gao and R. Hutton, Phys. Rev. A 50, 2313 (1994).

[15] H. F. Krause, et al., Abstracts of Contributed Papers, Nineteenth International Conference on 
the Physics of Electronic and Atomic Collisions (ICPEAC), July 1995, Eds. J.B.A. Mitchell, et al., 818 (1995).

[16] J.H. Derrickson, P.B Eby, K.H. Moon, T.A. Parnell, D.T. King, J.C. Gregory, Y. Takahashi, T. Ogata, Phys. Rev. A 51, 1253 (1995).

[17] N. Clayton, A. Belkacem, T. Dinneen, B. Feinberg, and H. Gould, Phys. Rev. A 55, R842 (1997).

[18] C. Bottcher and M.R. Strayer, Phys. Rev. D 39, 1330 (1989).

[19] J.-S. Wu, C. Bottcher, and M. R. Strayer, Phys. Lett. B 252, 37 (1990).

[20] J. Eichler, Phys. Rev. Lett. 75, 3653 (1995); D. C. Ionescu, J. Eichler, Phys. Rev. A 54, 4960 (1996)

[21] K. Momberger, A. Belkacem, A.H. Sorensen, Phys. Rev. A 53, 1605 (1996).

[22] G. Baur, Phys. Rev. A 42, 5736 (1990).

[23] M.J. Rhoades-Brown and J. Weneser, Phys. Rev. A 44, 330 (1991).

[24] A.J. Baltz, M. J. Rhoades-Brown, and J. Weneser, Phys. Rev. A 44, 5569 (1991); 47, 3444 (1993); 48, 2202 (1993); and 50, 4842 (1994).

[25] C. Best, W. Greiner and G. Soff, Phys. Rev. A 46, 261 (1992).

[26] D.C. Ionescu, Phys. Rev. A 49, 3188 (1994).

[27] K. Hencken, D. Trautmann, and G. Baur, Phys. Rev. A 51, 998 (1995); and 51, 1874 (1995).

[28] M.C. Guclu, J.C. Wells, A. S. Umar, M.R. Strayer and D. J. Ernst, Phys. Rev. A 51, 1836 (1995).

[29] A.J. Baltz, Phys. Rev. A 52, 4970 (1995).

[30] A.J. Baltz, M.J. Rhoades-Brown, and J. Weneser, Phys. Rev. E 54, 4233 (1996). 
[31] A. Alscher, K. Hencken, D. Trautmann, and G. Baur, Phys. Rev. A 55, 396 (1997).

[32] A.J. Baltz, Phys. Rev. Lett. 78, 1231 (1997).

[33] J.C. Wells, V.E. Oberacker, A.S. Umar, C. Bottcher, M.R. Strayer, J.-S. Wu, and G. Plunien, Phys. Rev. A 456296 (1992).

[34] R. Jackiw, D. Kabat, and M. Ortiz, Phys. Lett. B 277, 148 (1992).

[35] K. Rumrich, W. Greiner, and G. Soff, Phys. Lett. A 149, 17 (1990).

[36] K. Rumrich, G. Soff, and W. Greiner, Phys. Rev. A 47, 215 (1993).

[37] J. Eichler and A. Belkacem, Phys. Rev. A 54, 5427 (1996).

[38] N. Toshima and J. Eichler, Phys. Rev. A 42, 3896 (1990).

[39] I. Robinson and K. Rózga, J. Math. Phys., 25, 499 (1984).

[40] J.D. Bjorken, J.B. Kogut, and E. Soper, Phys. Rev. D 3, 1382 (1971).

[41] R. G. Newton, "Scattering Theory of Waves and Particles", (McGraw-Hill, New York, 1966).

[42] I.S. Gradshtein and I.M. Ryzhik, "Table of Integrals, Series, and Products", (Academic Press, San Diego, 1980).

[43] M. Abramowitz and I.A. Stegum, "Handbook of Mathematical Functions", (Dover Publications, New York, 1972). 


\section{FIGURES}

FIG. 1. Schematic diagram depicting a relativistic heavy-ion collision of two charges, $Z_{A}$ and $Z_{B}$, in the center-of-velocity frame with impact parameter $2 b$ and velocity $\beta$. Lorentz contraction is extreme, so the ions are depicted as oblate spheroids.

FIG. 2. Shown is the scalar component of the Lorentz-gauge interaction, $V^{0}$, for two different energies, (a) $\gamma=10$ (CERN-SPS energies), and (b) $\gamma=100$ (RHIC energies), plotted as a function of a narrow range of the $z$-coordinate for $t=0, \vec{b}=(1,0)$, and $\vec{r}_{\perp}=(2,0)$. Notice that away from the vicinity of $z=0$ this interaction is insensitive to the change in the energy of the ion.

FIG. 3. Same as Fig. 2, except that here the scalar component of the gauge-transformed interaction, $W^{0}$, is plotted. Notice that the gauge-transformed interaction is short-ranged, and that the range of the interaction decreases as the ion's energy increases.

FIG. 4. Schematic diagram of the time-history of a heavy-ion collision in the ultra-relativistic limit. Motion in the $t-z$ plane is shown with $\vec{r}_{\perp}$, and thus $\vec{b}$, assumed orthogonal to this plane. Ions $A$ and $B$ move toward each other in the $z$ direction with velocity $\beta=1$. The dotted lines are the projections of the ions trajectories on the $t-z$ plane, which for $\beta=1$ coincide with the intersection of this plane with the light fronts at $z= \pm t$.

FIG. 5. The light fronts, i.e. the hypersurfaces defined by $\tau_{ \pm}=0$, divide space-time into 4 distinct regions: (I) $\tau_{+}<0, \tau_{-}<0$; (II) $\tau_{+}>0, \tau_{-}<0$; (III) $\tau_{+}<0, \tau_{-}>0$; (IV) $\tau_{+}>0, \tau_{-}>0$.

FIG. 6. Depicted is the intersection of the surface $S$ enclosing region IV (shown as the broken line) with the $\tau_{+}-\tau_{-}$plane. Light-front components of the transition current, $J_{ \pm}$, are shown flowing into region IV at the light fronts, and the time-like component of the transition current, $J_{0}$, is shown flowing out of IV at the constant, large time $t_{f}$. 
FIG. 7. On the left are two terms of our result Eq. (69) for the amplitudes, indicated by their respective space-time maps: $\mathrm{I} \rightarrow \mathrm{II} \rightarrow \mathrm{IV}$, and $\mathrm{I} \rightarrow \mathrm{III} \rightarrow \mathrm{IV}$. On the right are shown the two Feynman diagrams of second-order perturbation theory [18] with their respective time ordering. Our result assumes large $\gamma$. Reference [18]assumes small $\alpha Z$. Exact agreement between the two results is obtained in the combined limit. 
Figure 1

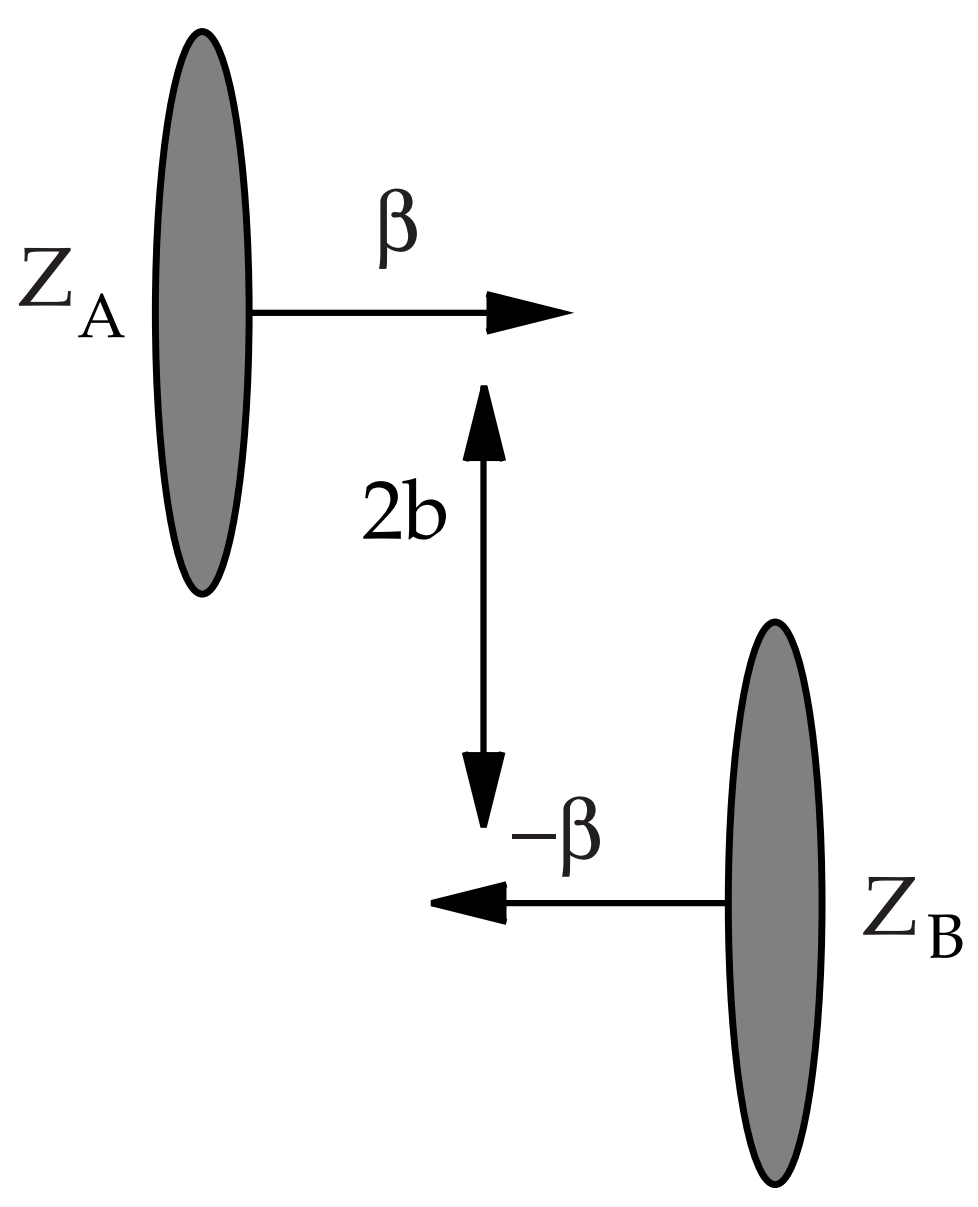


Figure 2

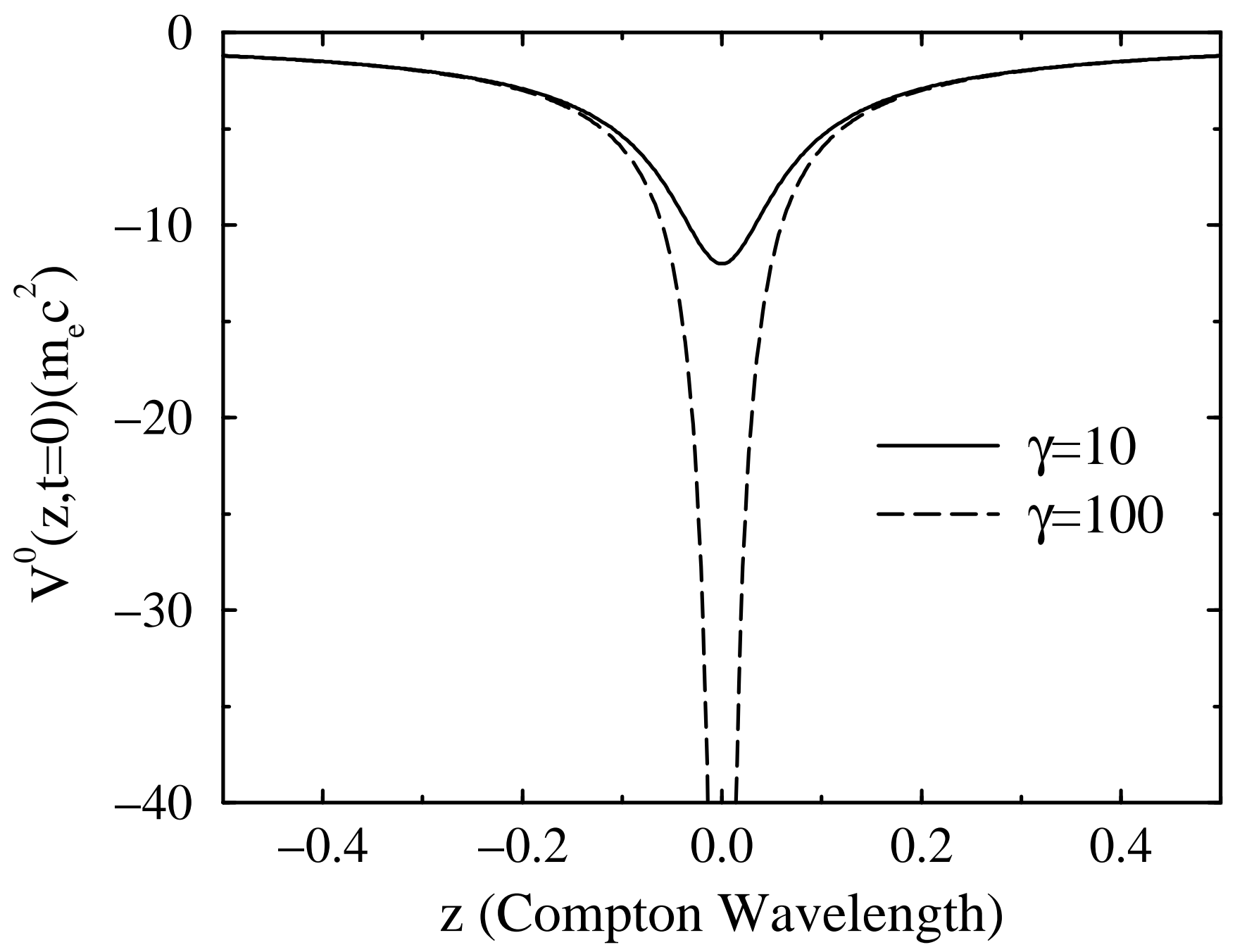


Figure 3

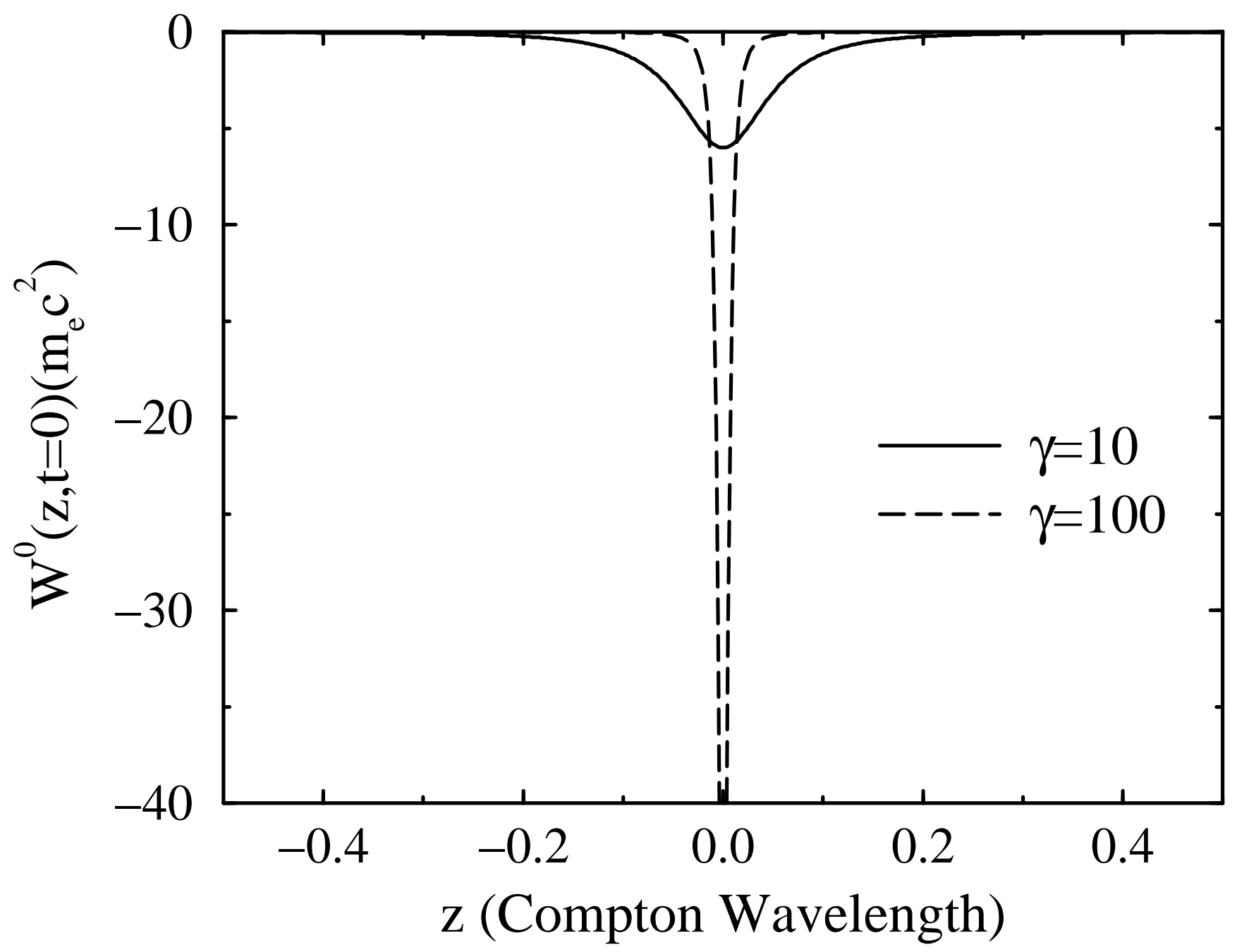


Figure 4, Segev and Wells

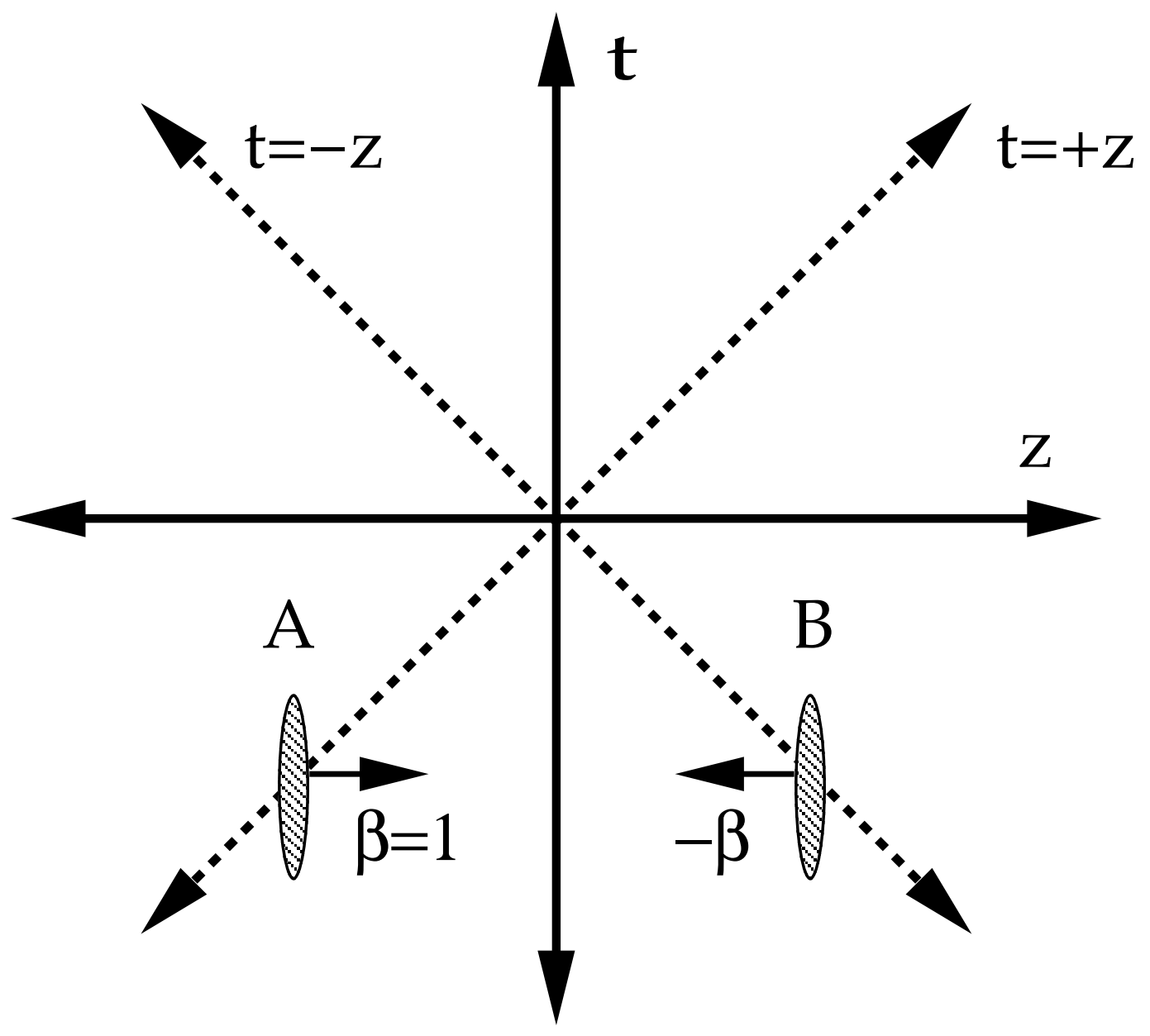




\section{Figure 5, Segev and Wells}

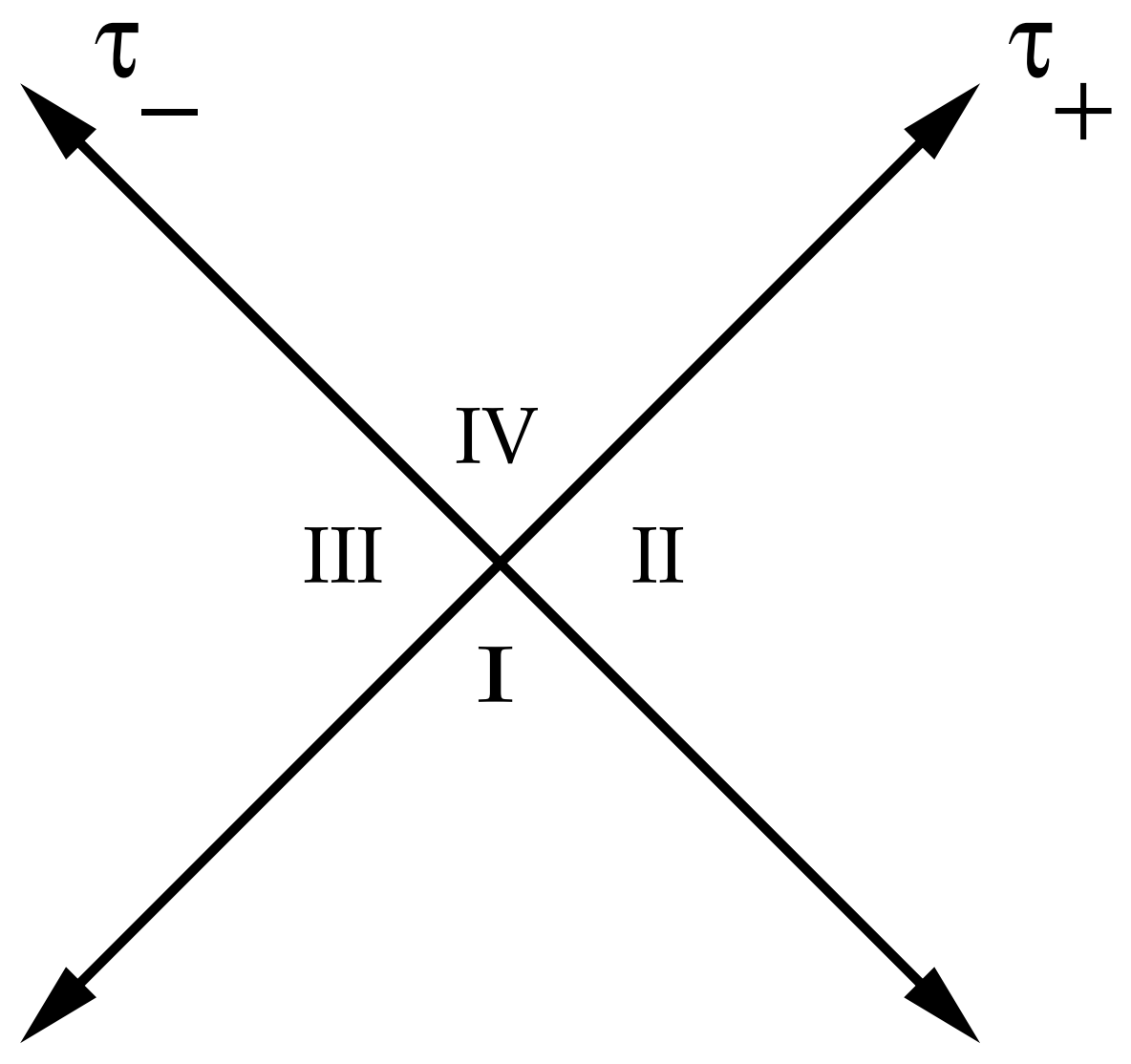


Figure 6, Segev and Wells

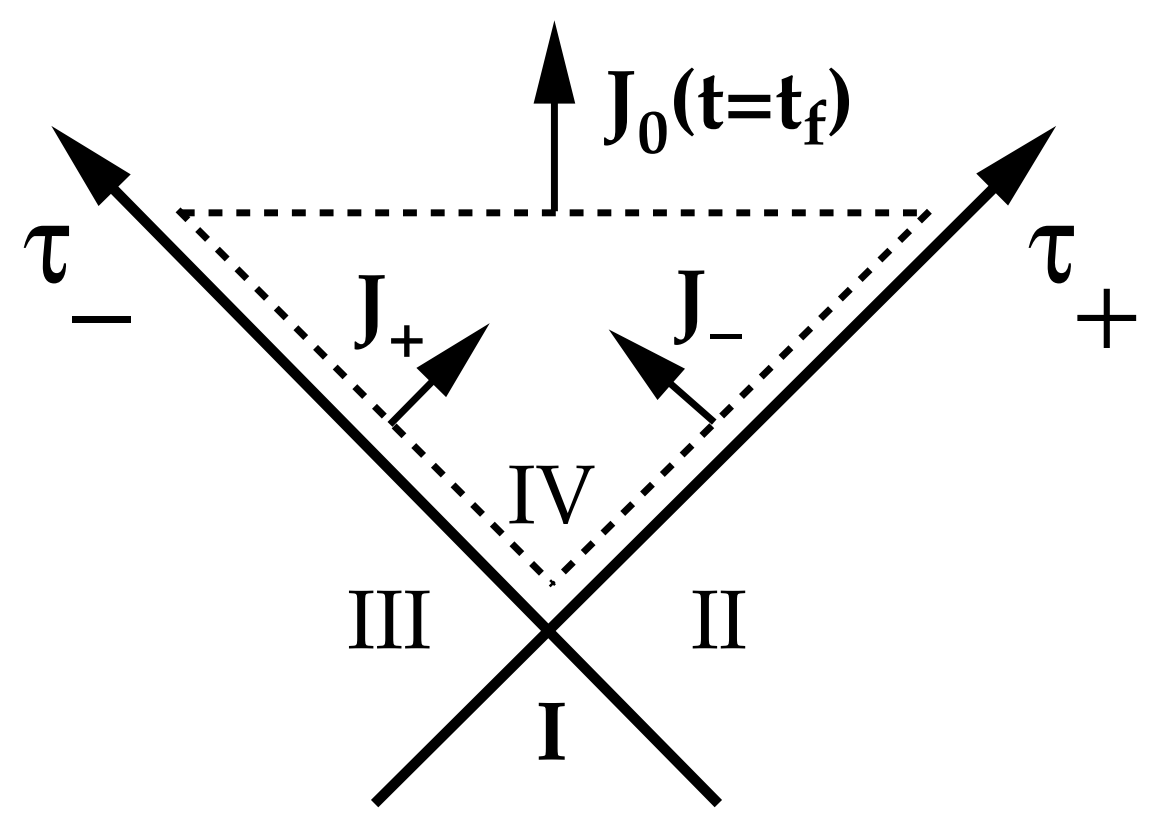


Figure 7, Segev and Wells
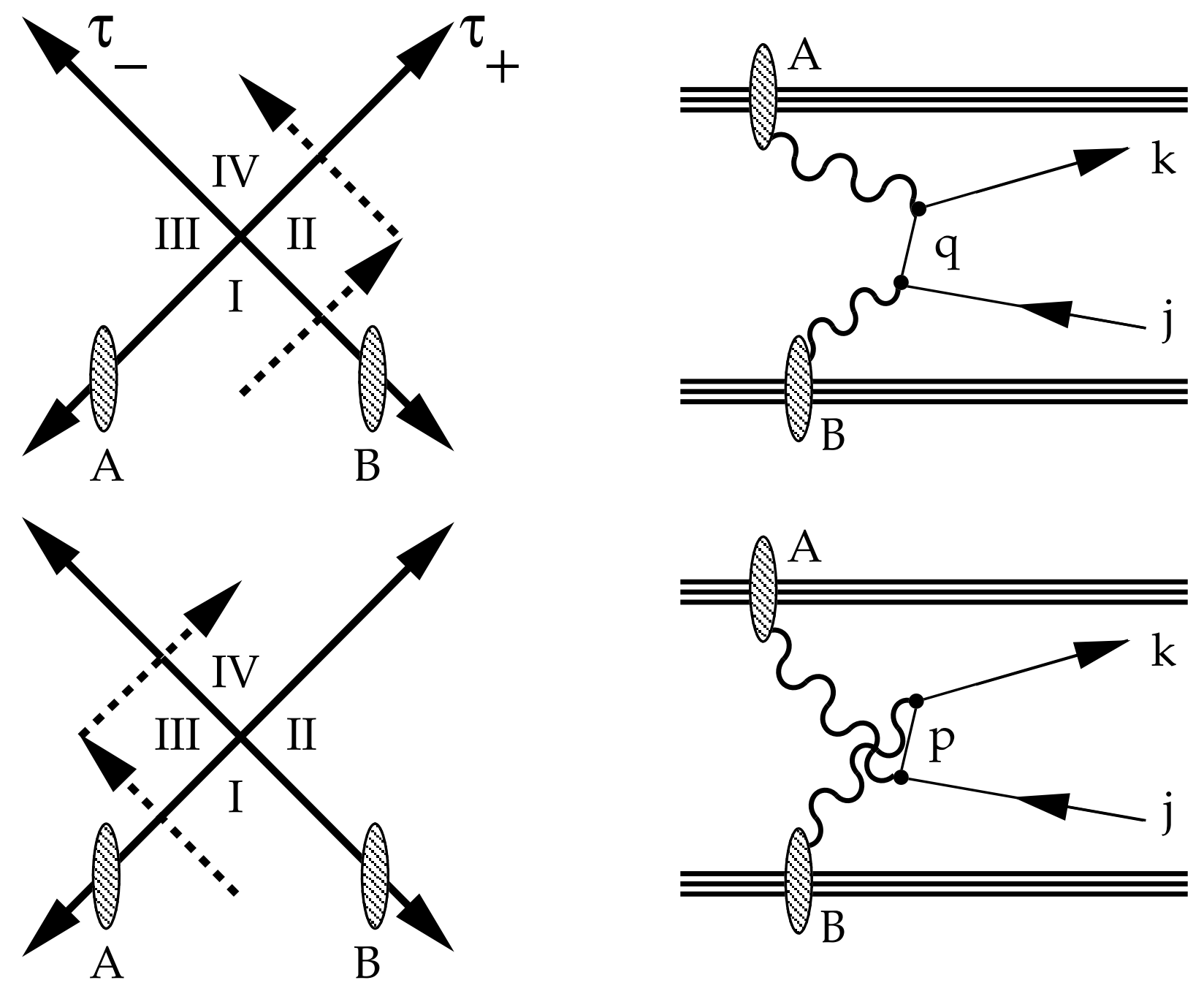